Review:

\title{
Molecular dynamics in polymeric systems
}

\author{
Madalena Dionísio ${ }^{1}$, Natália M. Alves ${ }^{2,3}$, João F. Mano ${ }^{2,3}$ *
}

${ }^{1}$ REQUIMTE/CQFB, Departamento de Química, FCT, Universidade Nova de Lisboa, 2829-516 Caparica, Portugal; Fax 212948550; Madalena.dionisio@dq.fct.unl.pt

${ }^{2}$ Departamento de Engenharia de Polímeros, Universidade do Minho, Campus de Azurém 4800-058 Guimarães, Portugal; Fax 253510339; jmano@dep.uminho.pt, nalves@dep.uminho.pt

${ }^{3}$ 3B's Research Group - Biomaterials, Biodegradables, Biomimetics, Universidade do Minho, Campus de Gualtar, 4710-053 Braga, Portugal

(Received: April 23, 2004; published: July 3, 2004)

\begin{abstract}
It is well known that the properties of polymeric materials depend strongly upon their chemical structure. Other more specific factors that may be related to the chemical structure also determine the macroscopic behaviour of such materials, namely the relative position of the different segments of the polymeric chain, the molecular architecture (molecular weight distribution, branching, copolymer organisation, cross-linking extent, etc.), the crystalline environment and the pressure/temperature conditions. All these factors have a common impact in the material: they are strongly correlated to the mobility on the molecular level. That is why a huge amount of work has been devoted to the study of translational/rotational mobility that occurs within the polymeric chains. This review is intended to provide a brief survey on such kinds of mobilities, how they can be studied and what are their main characteristics. Examples on systems studied in our groups will be provided, obtained by dielectric and mechanical spectroscopies and differential scanning calorimetry. It will be mainly focused on molecular motions that occur in the solid phase (i.e., to temperatures up to the rubbery plateau). The dynamics in blends or copolymers will be avoided here, as they would deserve a special discussion in their own context. Special attention will be paid to the glass transition and the mobility that occurs below and above it. The dynamics that are observed in peculiar systems, such as semi-crystalline or liquid crystalline polymers, will be addressed.
\end{abstract}

\section{Introduction}

Molecular dynamics observed in organic solids is usually characterised by both time scale and geometry. Geometry, or topology, is quantified in terms of parameters' order, amplitude of the motions, or number of sites that may be occupied by the molecular process. NMR is the most powerful technique used in such studies [1]. In this work, we will essentially address the description of the time scales of molecular processes. Those cover a broad frequency range, being associated with the length scale of the conformational mobility in the polymeric chain. Thus, the dynamic in polymeric materials involves a series (hierarchy) of relaxation processes that goes 
from very local motions $(\gamma, \delta, \beta \ldots$ processes), segmental mobility exhibiting co-operativity ( $\alpha$ relaxation), or even relaxation processes involving large or complete polymeric segments (Rouse dynamics or reptation). Fig. 1 intends to schematically depict such examples. The dynamics related to mobility shown in Fig. 1c is out of the scope of this review.
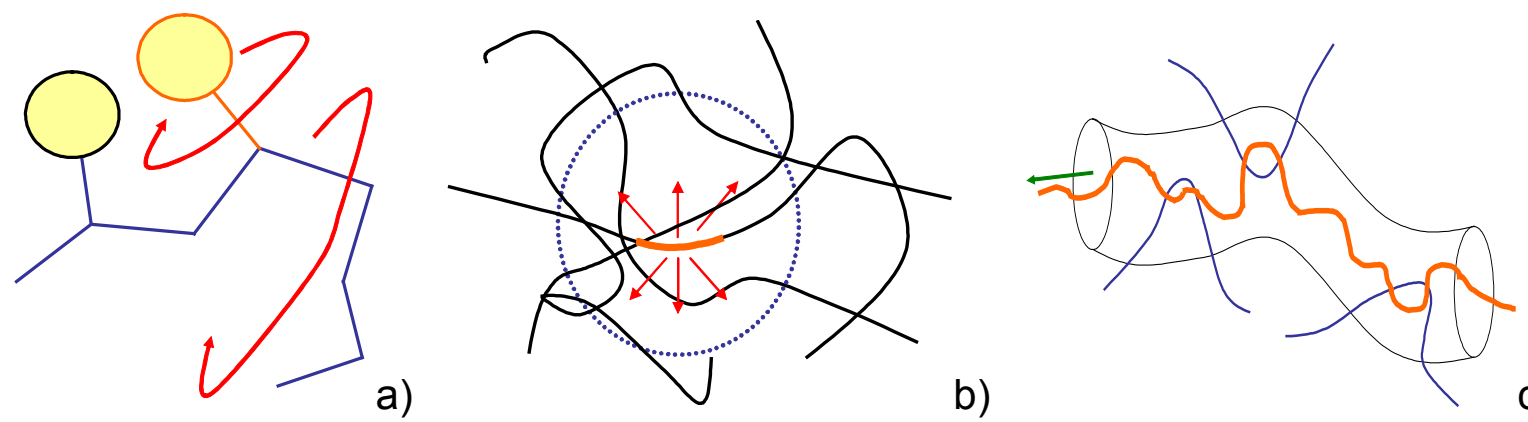

c)

Fig. 1. Molecular motions that may be found in polymeric systems: a) local mobility, assigned to relaxations occurring in the glassy state $(\gamma, \delta, \beta \ldots$ processes); b) cooperative segmental mobility, assigned to glass transition dynamics ( $\alpha$ relaxation); c) slower processes, attributed to motions of chain segments between entanglements

The structure of this work will follow an increasing temperature path. Thus, the local mobility, with shorter characteristic times, will be first discussed; then slower processes will be progressively addressed. Examples will be given, obtained by differential scanning calorimetry, and mainly by dielectric or mechanical spectroscopies. These are the most used techniques in the characterisation of molecular dynamics, the main features of which will be presented in the next section.

Spectroscopic techniques may cover large time/frequency ranges (e.g., dielectric spectroscopy equipments may cover 18 decades of frequencies) and are routinely used in many laboratories. However, their main drawback is the impossibility to attribute a given relaxation to a specific motion within the polymeric chain, or to inform about the geometry of the dynamic process, i.e., they lack molecular resolution. This information may be obtained by solid-state NMR that, however, works within a much narrower frequency range (typically between $1 \mathrm{~ms}$ and $1 \mathrm{~s}$ ), besides the complexity of the data treatment. The ideal situation to gain a full picture of the molecular dynamics would be the combination of both methods.

\section{Techniques used in the study of relaxational processes}

A variety of spectroscopic techniques have been used to study relaxational phenomena in polymeric systems [2-5] and among them, those using a cyclic electric (dielectric relaxation spectroscopy) or mechanical (dynamic mechanical analysis) stimulus are the most frequently used. Both techniques allow studying in a detailed way the distinct types of molecular mobility that occur in a given material for a large temperature and time scale.

Dynamic mechanical analysis (DMA) measures the dynamic mechanical properties of a given material, namely the two components of the complex modulus or complex compliance and the damping factor. A sinusoidal varying stress (strain) is applied and the resulting sinusoidal strain (stress) is measured as a function of temperature or 
frequency (time). This technique can be applied to study any material. As polymers are viscoelastic to a greater or lesser extent according to the experimental conditions at which they are measured, this technique is particularly useful in their characterization. Dielectric relaxation spectroscopy (DRS) measures the dielectric properties of a material, namely the dielectric constant and the dielectric loss as a function of temperature or frequency (time), when an oscillatory electrical field is applied. Its application is limited to materials containing polar groups/moieties.

Either in the mechanical or electrical case there is a stimulus, represented by the mechanical stress, $\sigma$, or the electric field, $\boldsymbol{E}$, which leads to a response, as given by the strain, $\varepsilon$, or the dielectric displacement, $\boldsymbol{D}$ (related to the polarisation, $\boldsymbol{P}$, by $\boldsymbol{D}=\boldsymbol{E}$ $+4 \pi P)$. In all the cases, the stimulus and the response are related by a linear equation. To describe the general situation a function called the primary response function is used. It is introduced by considering the effect of an infinitely short pulse, as represented by $\varphi(t)=\varphi_{0} \delta(t)$, where $\delta(t)$ is the delta function (which is zero for all values of time except for $t$, where it diverges). The primary response function $\mu(t)$ describes the time-dependent response, also called generally 'displacement' for the mechanical and electrical case, $x(t)$, caused by this pulse: $x(t)=\varphi_{0} \mu(t)$, allowing to establish a general expression between the response and an arbitrary applied timedependent stimulus:

$$
x(t)=\int_{-\infty}^{t} \mu\left(t-t^{\prime}\right) \varphi\left(t^{\prime}\right) \mathrm{d} t^{\prime}
$$

This expression is a consequence of the application of the superposition principle for linear systems. Taking into account this principle, an arbitrary time-dependent stimulus can be divided into a sequence of pulses with adjusted heights, and the total reaction is just the sum of the responses to all the pulses.

Using the previous expression it is now possible to derive the relations between distinct response functions, which are formulated in terms of the general variables $x$ and $\varphi$. To illustrate this general description, the example of a dynamic experiment (either mechanical or dielectric) is given. The application of an oscillatory stimulus $\varphi(t)=\varphi_{0} \exp (\mathrm{i} \omega t)$ leads to a 'displacement' $x(t)=x_{0} \exp (-\mathrm{i} \delta) \exp (\mathrm{i} \omega t)$. Applying Eq. (1) leads to:

$$
\frac{x_{0} \exp (-\mathrm{i} \delta) \exp (\mathrm{i} \omega t)}{\varphi_{0}}=\int_{-\infty}^{t} \mu\left(t-t^{\prime}\right) \exp \left(\mathrm{i} \omega t^{\prime}\right) \mathrm{d} t^{\prime}
$$

The ratio $\frac{x_{0} \exp (-\mathrm{i} \delta)}{\varphi_{0}}$, which appears in Eq. (2), describes this kind of experiments and is called general dynamic susceptibility, $\alpha^{*}(\omega) . \alpha^{*}(\omega)$ and $\mu(t)$ are related by the Fourier transform function:

$$
\alpha^{*}(\omega)=\int_{-\infty}^{t} \mu\left(t-t^{\prime}\right) \exp \left[\mathrm{i} \omega\left(t-t^{\prime}\right)\right] \mathrm{d} t^{\prime}
$$

It can be shown, by calculating the work that results from the displacement under the action of the stimulus, that $\alpha^{*}(\omega)$ has two components, $\alpha^{*}(\omega)=\alpha^{\prime}(\omega)-i \alpha^{\prime \prime}(\omega)$. $\alpha^{\prime}(\omega)$ is proportional to the reversible exchanged work, being a measure of the stored energy, whereas $\alpha "(\omega)$ is a measure of the dissipated energy during the process. For the mechanical and dielectric cases the dynamic susceptibility $\alpha^{*}(\omega)$ can be identified 
with the complex mechanical compliance $\left(J^{*}(\omega)=\varepsilon(t) / \sigma(t)\right)$ and the complex dielectric permittivity $\left(\varepsilon^{*}(\omega)=\boldsymbol{D}(t) / \boldsymbol{E}(t)\right)$, respectively. The typical variation of $\alpha^{\prime}(\omega)$ and $\alpha^{\prime \prime}(\omega)$ as functions of either frequency (at constant temperature) or temperature (at constant frequency) is presented in Fig. 2, for a single-time relaxation process. The $\alpha$ " $v s$. log $\omega$ plot is usually referred to as loss peak or dispersion curve. The maxima in the dispersion curve occur when the time scale of the experiment is of the same magnitude as the time scale of the molecular motions, $\tau(T)$.
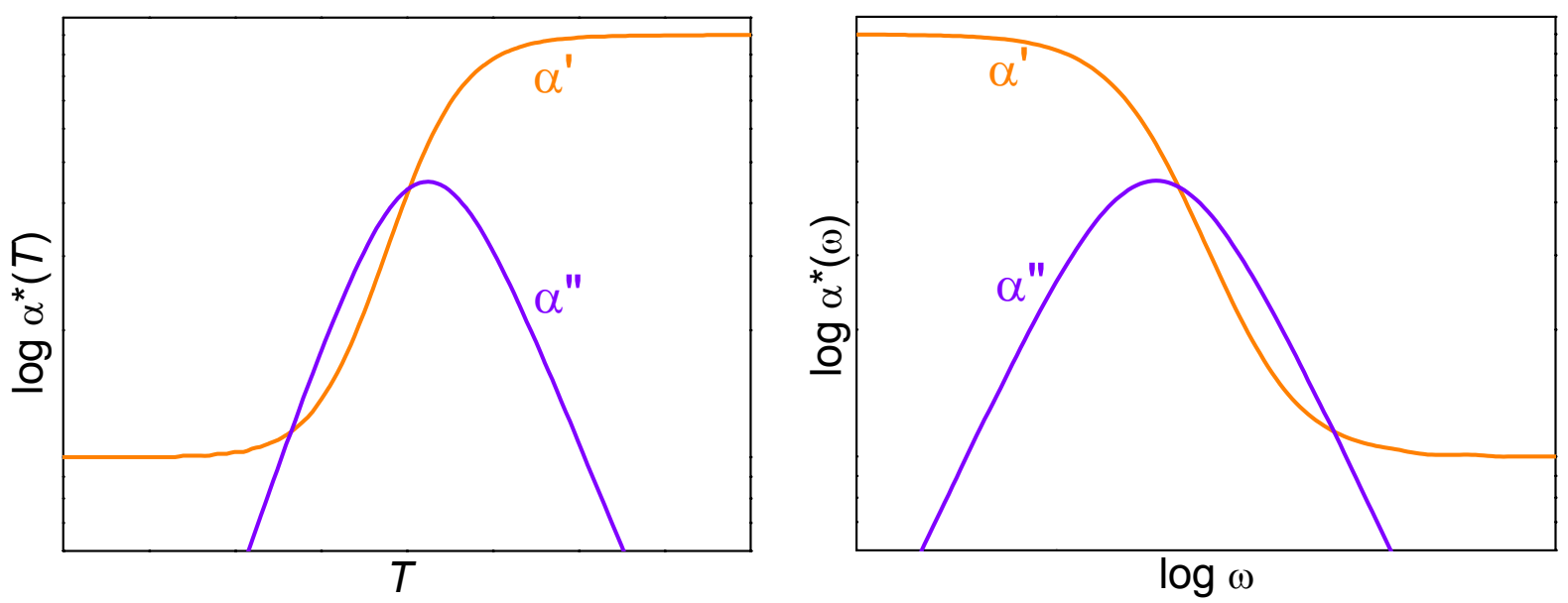

Fig. 2. Typical variation of $\alpha^{\prime}(\omega)$ and $\alpha^{\prime \prime}(\omega)$ for a Debye system (single relaxation time), as a function of frequency and temperature

When it is studied on a large frequency/time scale, the response of a given material under a dynamic stimulus usually exhibits several relaxations, as referred to in the Introduction, and not only one, as depicted in Fig. 2. Moreover, the loss peaks are usually broad and, sometimes, are associated to superposed processes. The magnitude of the variation in $\alpha^{\prime}(\omega)$, the breadth and intensity of the loss peak depend on the motions associated to a given relaxation. In general, the same relaxation/retardation processes are responsible for the mechanical and dielectric dispersions observed in polar materials, although some rearrangement processes could originate a stronger dielectric effect and vice-versa. Of course, in materials with low polarity the dielectric relaxations are very weak and not easily detected. The main relaxation processes observed in polymeric systems will be analysed in the next sections.

It must be pointed that, besides the example given here for the mechanical and electrical experiments, many other effects represent linear responses, too. For example, the application of a magnetic field that induces a magnetization or even the response of a differential scanning calorimeter (DSC), where the stimulus is the temperature $(T)$, the heat ( $T \mathrm{~d} S$, being $S$ the entropy) represents the response and the specific heat capacity $\left(C_{p}=T \mathrm{~d} S / \mathrm{d} T\right)$ can be viewed as a susceptibility.

\section{3. $\beta$ Relaxation}

Localized motions, involving either in-chain movements or side groups laterally attached to the main chain, are in the origin of the secondary relaxation processes. This type of local dynamics remains active even when the polymer is in the glassy state, i.e., when the large length scale backbone motions are frozen. At a fixed 
frequency, the $\beta$ relaxation is located at the highest temperatures in comparison with the other sub-glass processes, being preceded, respectively, by the $\delta$ and $\gamma$ relaxations with even more restricted dynamics and shorter relaxation times, in the order of decreasing temperature (or in the order of increasing frequency for a fixed temperature).

The $\beta$ relaxation is attributed to local motions such as hindered rotations of side groups (or of its subunits) that can occur independently of the backbone movements, conformational changes in cyclic side groups [3] or limited motions within the main chain (see Fig. 1). Nevertheless, the molecular origin of the $\beta$ relaxation is not completely understood [6], being observed in a variety of materials other than polymers, including glass-forming liquids and rigid molecular glasses, seeming a general feature of the amorphous state as proposed by Goldstein and Johari [7]. Theoretical developments [8] within the so-called coupling model introduced by Ngai and co-workers $[9,10]$ seem to point in the direction of a universal slow $\beta$ relaxation closely connected to the glass transition process (in this context the term 'slow' is to distinguish from 'fast' $\beta$ processes, predicted by the coupling model theory, ascribed to fast relaxations and observed in a variety of glass formers in the $\mathrm{GHz}$ region ref. [11] and refs. therein).

The $\beta$ relaxation is a thermally activated process, therefore, the temperature dependence of the relaxation time is Arrhenian-type, i.e., $\tau=\tau_{0} \exp \left(E_{\mathrm{a}} / R T\right) ; \tau_{\mathrm{o}}$ is the relaxation time at infinite temperature that, for a Debye process, is of the order of $10^{-12}$ $10^{-14} \mathrm{~s}$, and $E_{\mathrm{a}}$ is the activation energy that usually varies between 5 and $15 \mathrm{kcal} / \mathrm{mol}$, representing the potential barrier between two possible equilibrium states, as two different orientations of a polar side-group relatively to the main chain. $E_{\mathrm{a}}$ depends also on the environment of the group that undergoes conformational changes.

The above values of $\tau_{\mathrm{o}}$ and $E_{\mathrm{a}}$ are associated with strictly local processes due to individual mobility of sub-units, nearly with no activation entropy, according to the Eyring [12] formalism. $\tau_{0}$ values lower than $\approx 10^{-16} \mathrm{~s}\left(10^{-18}-10^{-40} \mathrm{~s}\right)$, imply an activation entropy greater than zero, being associated to more complex mobility mechanisms for which the activation energy could be of the order of $40-50 \mathrm{kcal} / \mathrm{mol}$, as is the case of the $\beta$ relaxation either in polysaccharides [13,14], and in polymers where the conformational mobility of the main chain is severely restricted [15].

As previously mentioned, the molecular mobility can be explored by dielectric relaxation spectroscopy, the dipole moment acting as a probe of the molecular motions, where the $\beta$ process results from local fluctuations of the dipole vector. The dielectric loss peak of this secondary relaxation is extremely broad due to a variety of molecular environments (structural heterogeneity) of the relaxing unity, and, consequently, a variety of energy barriers.

Fig. 3 shows the temperature dependence of the dielectric loss at $1 \mathrm{kHz}$ (in logarithmic scale) for poly(vinyl acetate). The $\beta$ process is located at the lowest temperatures (the $\alpha$ process comes out at higher temperatures). The inset illustrates the broadness and symmetry of this secondary process. The dielectric strength, which is proportional to the area under the loss peak, is much lower for the $\beta$ process relatively to the $\alpha$ relaxation, analysed in the next section, being the reason why loss data were plotted in logarithmic scale. This is a common pattern found in both polymer materials and glass formers. The secondary process is even more depleted in linear polymers that contain the dipole moment rigidly attached to the main chain, such as poly(vinyl chloride) [16] and polycarbonate [17-19]. 


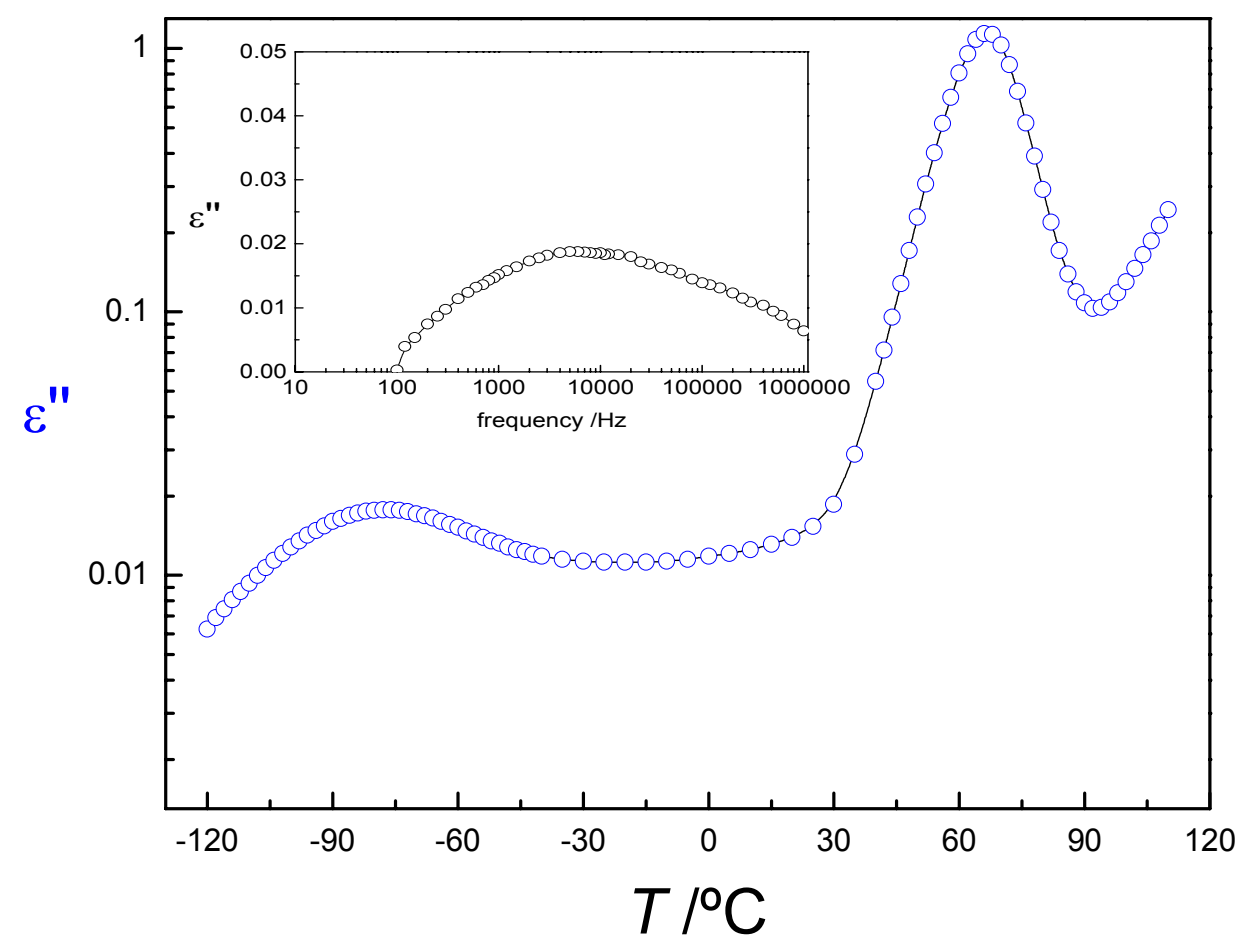

Fig. 3. Dielectric loss at $1 \mathrm{kHz}$ (in logarithmic scale) as a function of temperature for poly(vinyl acetate), showing at the lowest temperatures the secondary $\beta$ process and at the highest temperatures the $\alpha$ process. The inset shows the isothermal plot of the loss factor at $-60^{\circ} \mathrm{C}$ illustrating the broadness of the $\beta$ relaxation

Polymers with flexible polar side-groups, like poly(n-alkyl methacrylate)s, constitute a special class where the $\beta$ relaxation is rather intense, since it involves a $\pi$ flip of the side group around the bond that links it to the main chain. NMR studies demonstrate, for this type of polymers, that the motion of the side group is coupled with the main chain motions, determining the type of $\alpha \beta$ coupling (this issue will be described in section 6).

\section{Physical ageing}

\subsection{General features}

As mentioned before, this discussion of molecular mobility and relaxational processes is presented in the order of increasing temperature. Still related to the dynamics below $T_{\mathrm{g}}$, but near this temperature, large-scale mobility can occur besides the local mobility described in the previous section. This process, designated as physical ageing or structural relaxation [20-22], is related to the metastable glassy phase. The most common situation in which physical ageing is studied is when a material is cooled from above its $T_{\mathrm{g}}$ to an ageing temperature, $T_{\mathrm{a}}$, below $T_{\mathrm{g}}$. Thereafter, if the material is kept for a given time $\left(t_{\mathrm{a}}\right)$ at $T_{\mathrm{a}}$, its state will approach the equilibrium. Ageing is then manifest as, e.g., an apparent shift of the creep response to longer times or a reduction in the specific volume $V$ or enthalpy $H$, as illustrated in Fig. 4a. In this example it is seen how isothermal annealing at $T_{\mathrm{a}}$ reduces $H$, this effect becoming more pronounced as $t_{\mathrm{a}}$ increases. So, physical ageing, which can simply be defined as the material's relaxation toward equilibrium, is a kinetic pheno- 
menon that results from the non-equilibrium state characteristic of the glassy state. Glass transition is also a kinetic phenomenon and the glass transition temperature, $T_{\mathrm{g}}$, depends on the experimental time scale. $T_{\mathrm{g}}$ is usually defined as the temperature at which the relaxation time is about $100 \mathrm{~s}$ or the viscosity reaches the value of $10^{13}$ Poise. Another common definition is the calorimetric $T_{\mathrm{g}}$, measured by DSC (typically at $10^{\circ} \mathrm{C} / \mathrm{min}$ ) and defined as the midpoint of the specific heat capacity increment at the glass transition.

It is generally accepted that the main properties of physical ageing, also called structural relaxation, are non-linearity and non-exponentiality [20-22]. The nonlinearity is revealed by the asymmetry of the approach to equilibrium. Some of the best examples of this characteristic can be found in the pioneering work of Kovacs about volume relaxation [23]. If one jumps from $T_{0}=T-\Delta T$ or $T_{0}=T+\Delta T$ to $T$, one finds that the response from below $T$ is significantly different from that when the jump is from above $T$. This occurs as a result of the dependence of the relaxation time on both the temperature and the structure of the glass. The non-exponentiality is a consequence of the existence of a distribution of relaxation times. The evidence of the existence of this distribution was also demonstrated by Kovacs with the so-called 'memory effects' [15], which show that the response of the glass is a function of the whole of its previous thermal and mechanical history since its most recent equilibrium state.
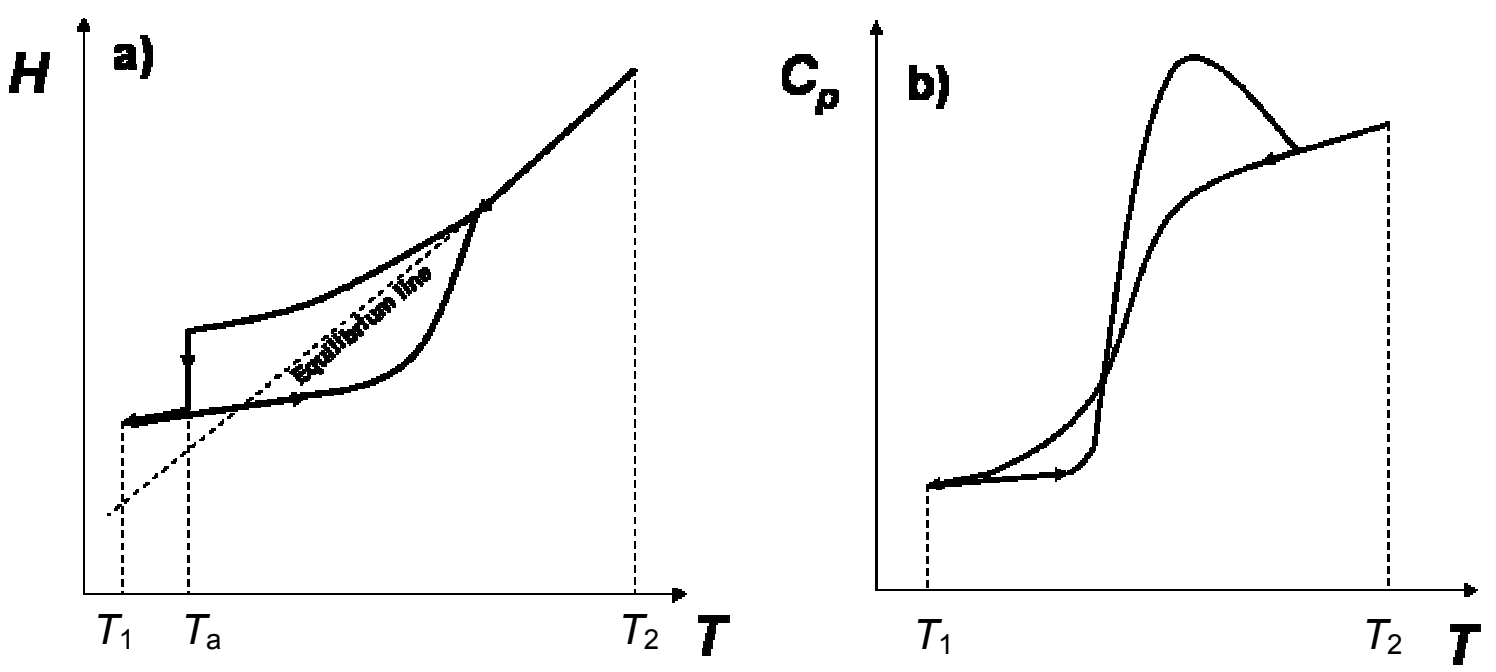

Fig. 4. Schematic representation of the evolution of a) enthalpy (or volume or entropy) and b) specific heat capacity during a cooling scan through the glass transition and a heating scan performed after ageing at a temperature $T_{\mathrm{a}}$ close to $T_{\mathrm{g}}$. (The diagonal line in Fig. $4 a$ is the equilibrium line)

Differential scanning calorimetry (DSC) is one of the most frequently used experimental techniques for the study of structural relaxation, where the property used to investigate structural relaxation is the enthalpy. In this case, this process is also referred to as enthalpic relaxation. By DSC one can measure the heat capacity $C_{p}$ as a function of temperature (Fig. $4 \mathrm{~b}$ ) and the enthalpy changes with ageing can be obtained by integration of the $C_{p}$ curves (Fig. 4a). The usual procedure is to perform a scan at constant heating rate from the aged glassy state $\left(T_{1}\right)$ to a temperature $T_{2}$ above $T_{\mathrm{g}}$. Then a second scan from an unaged state obtained immediately after the cooling stage is a reference for evaluating the effects of ageing at $T_{\mathrm{a}}$. The aged 
material usually presents a more or less pronounced endothermic peak in the glass transition region. The enthalpy loss between the aged and unaged states can be calculated from the difference between the areas under these two curves.

The application of the same procedure for distinct thermal histories (distinct $T_{\mathrm{a}}$ and $t_{\mathrm{a}}$ ) will provide a picture of the structural relaxation kinetics for a particular material. Of course the effect of just varying the cooling rate (from quenching to extremely low rates) can also be applied to the analysis of structural relaxation.

An important feature of physical ageing is that the ageing kinetics of a particular material is different for distinct properties, i.e., changes in one property cannot be predicted by using parameters from another property. This can be seen, e.g., in the work of Perez et al. [24], where the ageing of volume, enthalpy and dynamic mechanical properties of PMMA was compared. They found that the time scales for the evolution of these properties increased in the order mechanical < volume < enthalpy. Sasabe and Moynihan [25] found for PVC the same result as Perez et al. [24].

\subsection{Phenomenological models}

The study of structural relaxation may provide a deeper insight into the phenomenology of the conformational mobility of amorphous chains. Due to the nonlinearity and non-exponentiality of the structural relaxation as mentioned before, there is not a simple relationship between the measurable properties and the variables characterising the kinetics of the process, such as the relaxation time. Nevertheless, much effort has been made to develop mathematical models that permit the comparison of experimental $C_{p}$ curves obtained after distinct thermal histories with theoretical predictions. It has been shown that the main features of the structural relaxation process can be modelled on the basis of a distribution of relaxation times that depends both on the temperature and on the structure of the material represented by the value of the relaxing variable (in DSC experiments enthalpy or entropy). This type of models allows determining a series of parameters than can be in some way related to molecular mobility and it is also possible to accede to the relaxation times of the conformational rearrangements.

Narayanaswamy [26] was the first to show that non-linearity could be incorporated in the analysis of complex thermal histories. This involved the use of the reduced time $\xi$ that is a function of both temperature and separation from equilibrium:

$\xi=\int_{0}^{t} \frac{\mathrm{d} t^{\prime}}{\tau\left(t^{\prime}\right)}$

In most of the models, the non-exponential character of structural relaxation is described by the KWW equation [27], a function widely used for representing viscoelastic and dielectric relaxational processes:

$$
\phi(t)=\exp \left[-\left(\frac{t}{\tau}\right)^{\beta_{\mathrm{kWw}}}\right]
$$

The $\beta_{\mathrm{K} w w}$ parameter is associated with the distribution of characteristic times. A small value of $\beta_{\mathrm{kw} w}$ implies a broad distribution and $\beta_{\mathrm{kw} w}=1$ implies a narrow distribution (an exponential response).

The Narayanaswamy-Moynihan (NM) [28] and Scherer-Hodge (SH) models are the best known models [29]. In both models the dependence of the relaxation time $\tau(t)$ 
with structure relies on Tool's concept of a fictive temperature $T_{f}$ [30]. $T_{f}$ can be defined as the temperature at which the glass would have its equilibrium enthalpy value if it was instantaneously brought to that temperature.

The expression for $\tau\left(T, T_{\mathrm{f}}\right)$ proposed by the NM model is [28]:

$$
\tau\left(T, T_{\mathrm{f}}\right)=A \exp \left(\frac{\Delta h^{*}}{R}\left(\frac{x}{T}+\frac{1-x}{T_{\mathrm{f}}}\right)\right)
$$

$A$ is a constant, $R$ is the ideal gas constant and $x$ is a parameter between 0 and 1 that defines the relative contributions of temperature and structure to the relaxation time.

In the $\mathrm{SH}$ model an expression deduced from the Adam-Gibbs theory is applied [31], which relates the relaxation time to temperature and configurational entropy,

$$
\tau(T)=A \exp \left(\frac{B}{T S_{c}(T)}\right)
$$

and that leads to the following expression for $\tau\left(T, T_{\mathrm{f}}\right)$ :

$$
\tau\left(T, T_{\mathrm{f}}\right)=A \exp \left(\frac{D}{R T\left(1-T_{2} / T_{\mathrm{f}}\right)}\right)
$$

where $A$ and $D$ are constants and $T_{2}$ is the Gibbs-DiMarzio transition temperature [32].

Some years ago a new model was proposed by Gómez Ribelles and Monleón Pradas [33], the SC model, which introduced a new hypothesis related to the state attained at infinite time in the structural relaxation process at a temperature $T_{\mathrm{a}}$. One of the main assumptions in the models explained above is that an amorphous material kept in isothermal conditions in any out-of-equilibrium state would reach at infinite time an equilibrium state determined by the extrapolation to temperatures below $T_{\mathrm{g}}$ of the enthalpy equilibrium line corresponding to temperatures above the glass transition. This comes from the identification of the limit of the fictive temperature at infinite time with $T$. It has been proposed by the SC model that the limit at infinite time of the structural relaxation process could be a metastable state with higher configurational entropy and enthalpy than the equilibrium state obtained by extrapolation. By this way an additional parameter $\delta$ is introduced into the model, which defines the difference between the limit state that could be attained and the equilibrium state, besides the other four parameters: the pre-exponential constant $A$ and the parameter $B$ of the Adam-Gibbs Equation, the Gibbs-DiMarzio temperature $T_{2}$ and the exponent $\beta$ of the KWW equation. It has been shown that the agreement between model simulation and experiments is highly improved when the assumption of this metastable state is taken into account $[34,35]$.

An example of the application of the SC model to structural relaxation data of amorphous and semi-crystalline poly(ethylene terephthalate), PET [36], is given in Fig. 5. It can be seen that the fits are quite satisfactory for both materials, taking into account that heating scans corresponding to completely distinct thermal histories can be reproduced with the same set of parameters. From Fig. 5 it is also evident how the behaviour in the glass transition region is significantly different for amorphous and semi-crystalline PET. The influence of crystallinity in the glass transition dynamics will be discussed later. 

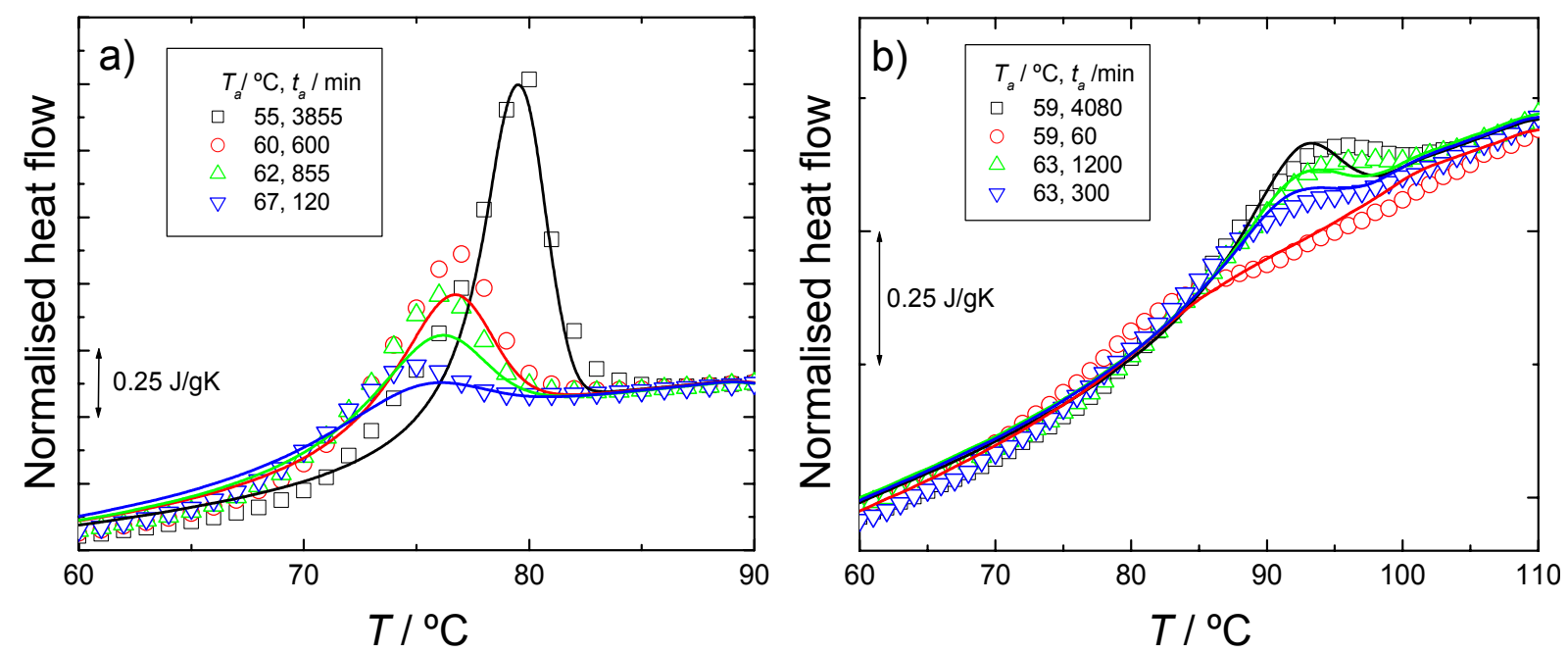

Fig. 5. Normalised heat flow as a function of temperature for a) amorphous PET and b) semi-crystalline PET, previously subjected to distinct thermal histories (indicated in the graphics). The lines represent the curves predicted according to the SC model. More details about experimental conditions and discussion of the results can be found in ref. [36]

As mentioned before, the use of phenomenological models enables to accede to the characteristic times of the conformational rearrangements. The variation of the mean relaxation time as a function of temperature $\tau(T)$ (for a given thermal history), obtained through application of the SC model, is presented in Fig. 6 for the semicrystalline PET of Fig. 5b. For the sake of comparison, the retardation times obtained from isothermal DMA experiments and the retardation times at the temperature of maximum strain rate $\tau\left(T_{\max }\right)$ calculated from the non-conventional thermally stimulated recovery (TSR) technique, on the same material, are also presented in Fig. 6. Related to TSR, it can be said briefly that it is an equivalent low-frequency technique, where the strain recovery of a sample, previously deformed under the application of a static stress, is monitored while the sample is subjected to an adequate temperature program, usually a heating at constant rate. This technique offers the interesting possibility of decomposing a complex process, characterised by a distribution of characteristic times, into narrow distributions of relaxations, by applying the thermal sampling procedure. It was shown that a commercial dynamic mechanical analyser can be used to perform TSR experiments $[37,38]$.

It can be seen that above $T_{\mathrm{g}}$ the DSC and DMA lines are quite parallel, the DSC times being higher than the DMA ones. As the same physical phenomenon (conformational rearrangements in the glass transition region) is analysed, it was expected that the evolution of the dynamics with temperature should be probed similarly by both techniques. However, as the measured properties and the type of solicitation are distinct, the DSC and DMA times at a given temperature shouldn't be necessarily the same, what in fact was observed. It should be mentioned that in the glassy state it is not correct to directly compare DSC and DMA data, because both experiments are performed under very distinct thermal histories. The DSC curve corresponds to a cooling experiment at $40^{\circ} \mathrm{C} / \mathrm{min}$ whereas the DMA data results from isothermal experiments, where the sample stayed $\approx 20 \mathrm{~min}$ at each temperature. The retardation times obtained by TSR are often a measure of the time scale of the experiments (in this case, thermal sampling experiments) due to the fact that at that temperature the variation of the strain during the recovery process is maximum. It is seen that the 
retardation times do not vary significantly in the logarithmic axis, taking values near $100 \mathrm{~s}$. The magnitude of these values is also typical of DSC time scales.

From differentiation of the DSC data it is also possible to obtain the variation of the apparent activation energy as a function of temperature. Fig. 7 compares the activation energies obtained by the three techniques for this material.

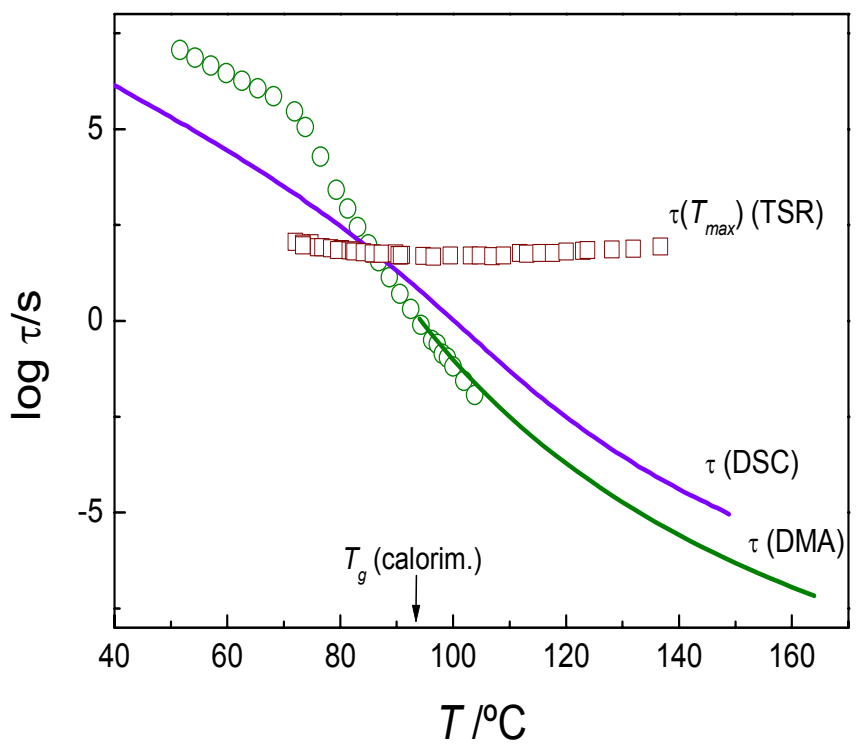

Fig. 6. Squares: retardation times at the temperature of maximum strain-rate $\left(T_{\max }\right)$ obtained from TSR experiments (thermal sampling procedure); violet line: mean characteristic time as a function of temperature obtained from DSC; circles: mean characteristic time as a function of temperature calculated from DMA experiments. The line linked to DMA data is an extrapolation to higher temperatures according to the VFTH equation. More details can be found in refs. $[36,39]$

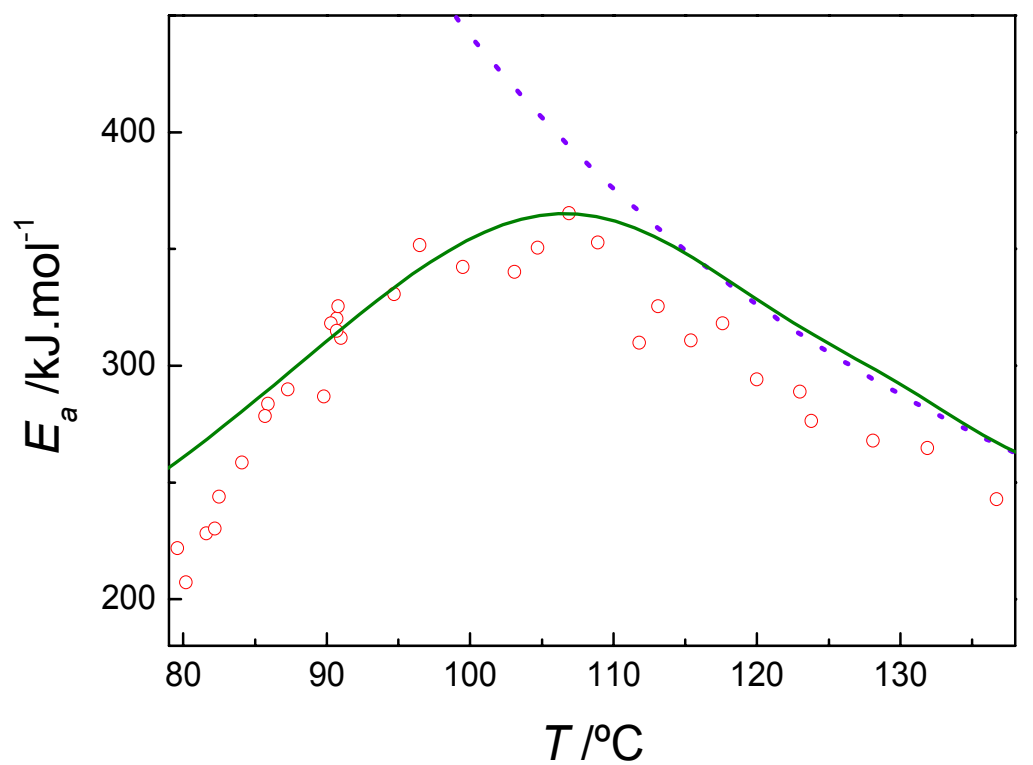

Fig. 7. Activation energy as a function of temperature calculated in both the glassy and equilibrium states from TSR (circles) and from DSC experiments (solid line). The dashed line is the activation energy calculated from the DMA results in the equilibrium state, using the VFTH equation. More details can be found in ref. [39] 
In this figure it can be seen that for $T>T_{\mathrm{g}}$ the $E_{\mathrm{a}}$ values obtained from all the techniques are very similar. Below $T_{g}$, a good agreement is found between DSC and TSR values. It can also be observed, either by DSC or TSR, how $E_{a}$ increases with temperature, reaching a maximum at a temperature around $T_{g}$, and then decreasing with temperature. This variation in the $E_{a}$ pattern corresponds to the transition between Arrhenius behaviour (characteristic of the metastable glassy state, whose mechanisms have been described in this section) and a typical behaviour of a liquid in equilibrium. The dynamics of segmental mobility above $T_{\mathrm{g}}$ has been successfully described by the VFTH or WLF equations, presented in the next section.

\section{5. $\alpha$ Relaxation}

The dielectric loss curves of the $\alpha$ relaxation are asymmetric and narrower than those of the $\beta$ relaxation. Fig. 8 illustrates this behaviour for poly(vinyl acetate). The $\alpha$ relaxation is associated to the glass transition phenomenon that, at a molecular level, remains an unsolved problem of the physics of condensed matter $[40,41]$. Nevertheless, it is well accepted that the dynamics at the glass transition is associated with the microbrownian segmental motions of chains (segmental mobility) [6,42], being cooperative in nature, which means that a specific segment moves together with its environment. Therefore, the $\alpha$ relaxation involves both intramolecular (connectivity within the main chain) and intermolecular (coordinated motion with the environment) interactions.

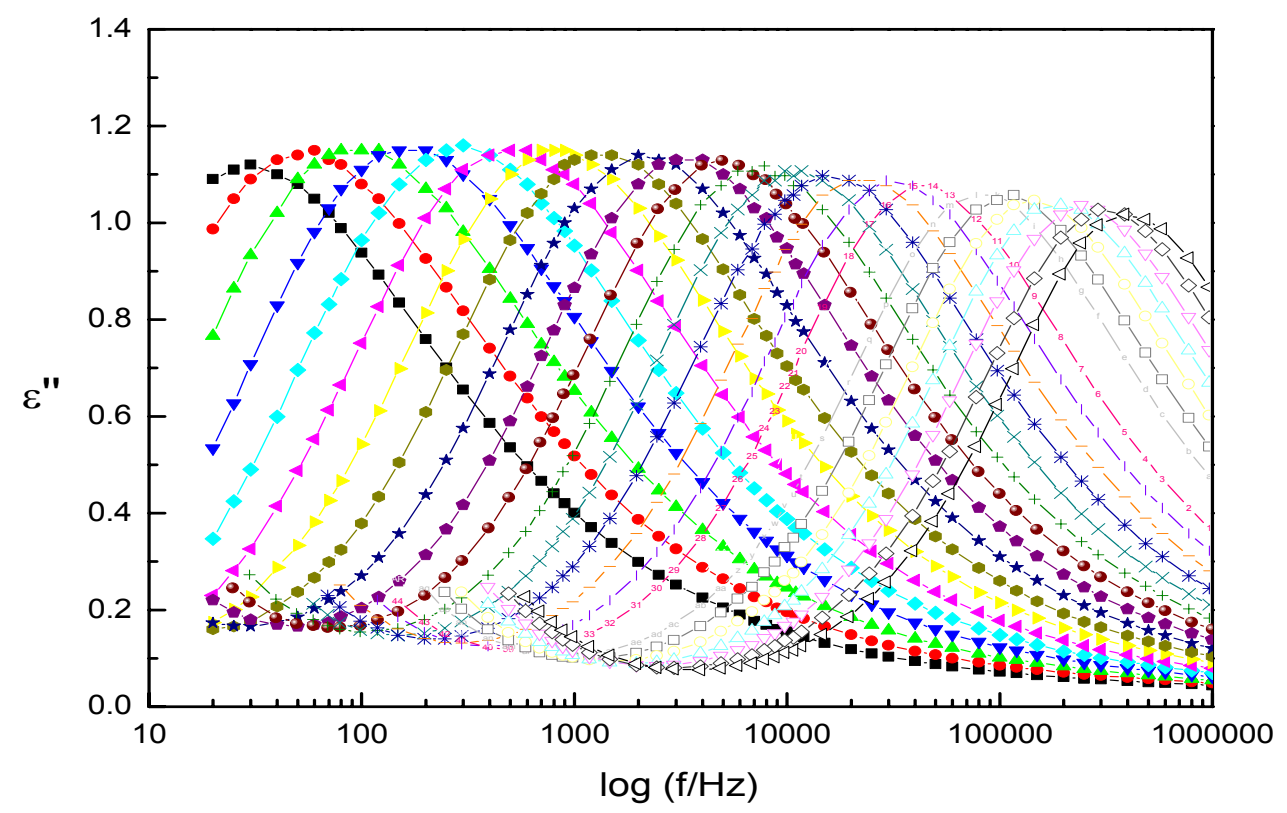

Fig. 8. Dielectric loss spectra of the $\alpha$ relaxation process of poly(vinyl acetate) between 54 and $110^{\circ} \mathrm{C}$

In the glass transition region the viscosity and, consequently, the relaxation time increase drastically as the temperature decreases. Thus, molecular dynamics is characterized by a wide distribution of relaxation times and a strong temperature dependence.

The changes in the relaxation time in the glass transition region could be interpreted as a change in the length scale of the segmental motions that are in the origin of the 
$\alpha$ process [42,43] that, close to $T_{\mathrm{g}}$, must be of the order of $2 \mathrm{~nm}$, increasing as the temperature decreases. This increase of the length scale with temperature decrease is associated with the cooperativity enhancement as described by Adam and Gibbs [31].

The logarithmic plot of the relaxation time as a function of the reciprocal of the temperature shows a departure from linearity, due to the abrupt increase of the relaxation time with the temperature decrease, thus presenting some curvature near $T_{g}$. This relaxation time dependence could be described by the Vogel-FulcherTammann-Hesse equation [44-46]:

$$
\ln \tau_{\alpha}=\ln \tau_{o}+\frac{A}{T-T_{o}}
$$

where $\tau_{\mathrm{o}}$ is of the order of $10^{-13} \mathrm{~s}$ and $T_{\mathrm{o}}$ is the Vogel temperature, interpreted as the glass transition temperature of an ideal glass, i.e., a glass obtained with an infinitely slow cooling rate; nevertheless the physical meaning of $T_{\mathrm{o}}$ is not completely clarified being described by different theories (see discussion in ref. [42]). This equation reverts to Eq. (7) when the system is in equilibrium, i.e., $T_{\mathrm{f}}=T$.

The VFTH equation can be rewritten as

$$
\ln \frac{\tau\left(T_{1}\right)}{\tau\left(T_{2}\right)}=\frac{-C_{1}\left(T_{1}-T_{2}\right)}{C_{2}+\left(T_{1}-T_{2}\right)}
$$

which is known as the Williams-Landel-Ferry (WLF) equation [47], where $C_{1}$ and $C_{2}$ are constants of the material, and where $\tau\left(T_{1}\right)$ and $\tau\left(T_{2}\right)$ are the relaxation times at, respectively, $T_{1}$ and $T_{2}$. This equation is supported by the free volume theory [48], where it is assumed that the molecular mobility at a specific temperature depends on the free-volume fraction at that particular temperature. It defines a temperature $T_{0}$ for which the free volume is zero. The WLF equation describes, for a wide variety of materials, the behaviour of the $\alpha$ relaxation process between $T_{\mathrm{g}}$ and $T_{\mathrm{g}}+100 \mathrm{~K}$ [3]. Experimental data obtained at a specific temperature can be superposed to experimental data obtained at a different temperature simply by a horizontal shift along the $\log t$ or $\log \omega$ axis - the time-temperature superposition principle. This procedure allows us to obtain so-called master or reduced curves for a specific material (as shown in ref. [49] for poly(vinyl acetate).

In the time domain, the $\alpha$ relaxation process of amorphous polymers is successfully described by the KWW function (Eq. (4)), with $\beta_{\mathrm{K} w w}$ values typically between 0.2 and 0.5. The disadvantage of this equation is its transformation into the frequency domain through a Fourier transform (Eq. (2)) that cannot be solved analytically (Koizumi and Kita [50] built a numerical table of complex permittivities for $\beta_{\mathrm{Kw} w}$ values in the range from 0.3 to 1). Therefore, one of the most popular equations in the literature is the Havriliak-Negami (HN) equation [51] (recently revised [52]):

$$
\varepsilon^{*}(\omega)=\varepsilon_{\infty}+\frac{\Delta \varepsilon_{j}}{\left[1+\left(i \omega / \omega_{o}\right)^{a}\right]^{b}}
$$

where $a$ and $b(0<a<1,0<a b<1)$ are shape parameters describing the slopes of the $\varepsilon$ " peak at frequencies lower and higher than $\omega_{0}$, that is the frequency of $\varepsilon$ "max: $a$ $=\partial \log \varepsilon " / \log \omega$ for $\omega<<\omega_{0}$, and $a b=-\partial \log \varepsilon " / \partial \log \omega$ for $\omega \gg \omega_{0}$ [53]; the value of $a$ is related to the peak width of the loss peak, and $b$ to its asymmetry (Debye behaviour corresponds to $a=a b=1$ ). This equation is an empirical modification of the Debye 
equation and, since it has two adjustable parameters, it describes with great success an isolated relaxation process (when the frequency window covers more than one process, the experimental data can be fitted with a sum of HN equations, one for each relaxation process detected). The classical Cole-Cole [54] (loss curves symmetrical about the position $\omega \tau=1$ ), and Cole-Davidson [55] (loss curves with high frequency broadening) equations are obtained, respectively, for $b=1$ and $a=1$.

Alegria and co-workers published a comparison between the $\mathrm{HN}$ and KWW descriptions $[53,56,57]$. The proposed empirical correlations allow to construct an analytical frequency domain function with a single shape parameter, which - in good approximation - is the Fourier transform of the KWW time decay function [57].

By both its simplicity and the way of rationalising the behaviour of all amorphous polymers [58] and glass formers [59], we also refer to the partial and total relaxation model of the dipole moment as proposed by Williams [42,60]. In this model it is assumed that in an amorphous material the dipoles exist in different local environments, partial relaxation occurring through localized mobility in a specific environment that originates the $\beta$ process. The dipole moment that persists at temperatures above the $\beta$ process is further relaxed by the microbrownian motions of the environment originating the $\alpha$ process or, at even higher temperatures, the a process that will be referred to in sections 6 and 7 .

The models therein presented, that attempt to describe the glass transition, are phenomenological in nature. A microscopic approach to the glass transition process was developed by Götze [61-63] through the 'mode coupling theory' (MCT), which explains the sequence of relaxation events that occur in a supercooled liquid in terms of a non-linear coupling between density fluctuation modes. At the glass transition, the system undergoes an ergodic-nonergodic transition at a well-defined critical temperature, $T_{\mathrm{c}}$.

\section{6. $\alpha \beta$ Splitting}

As mentioned before, the relaxation times of the $\alpha$ process show a strong temperature dependence, described by the VFTH equation (Eq. (9)), while the temperature dependence of the $\beta$ process follows Arrhenian behaviour. Therefore, in a logarithmic plot of the relaxation time vs. the reciprocal of temperature (activation plot), the $\alpha$ process corresponds to a curved line while the $\beta$ process is a straight line (Fig. 9). At temperatures well above $T_{\mathrm{g}}\left(1.2 T_{\mathrm{g}}-1.4 T_{\mathrm{g}}\right)$, the time scales of both relaxation processes tend to converge, since log $\omega_{\alpha}$ increases faster than log $\omega_{\beta}$ with the temperature increase. Consequently, the two lines in the activation plot come close together and eventually merge in a single process - the a process; or, with decreasing temperature, the a relaxation process bifurcates in two processes, $\alpha$ and $\beta$, and the frequency and temperature, $T_{\beta}$, region where the separation occurs is designated by ' $\alpha \beta$ splitting'. (Some authors use the designation ' $\alpha \beta$ process' that could lead to interpret this process as a simple superposition of both $\alpha$ and $\beta$ processes. As it will be shown later on, this is not true and thus the a designation is here adopted.) In polymers as polystyrene, poly(vinyl acetate) and poly(vinyl chloride), the $\alpha \beta$ splitting occurs in a frequency region between $10^{7}$ and $10^{10} \mathrm{~Hz}$ [65].

An interesting class of polymers, which present the time scales superposition associated to movements of both large and short length scale, is that of poly(n-alkyl methacrylate)s that have the general formula $-\left(\mathrm{CH}_{2}-\mathrm{C}\left(\mathrm{CH}_{3}\right)(\mathrm{COOR})\right)^{-}$, and in which 
molecular dynamics is strongly determined by the ester side group COOR and by the size of the alkyl group R. In this type of polymers a pronounced decrease of the glass transition temperature is observed with increasing size of the alkyl group, which is designated as an internal plasticization effect, being attributed to an increase in the distance between adjacent polymer chains that increases the mobility of the main chain.

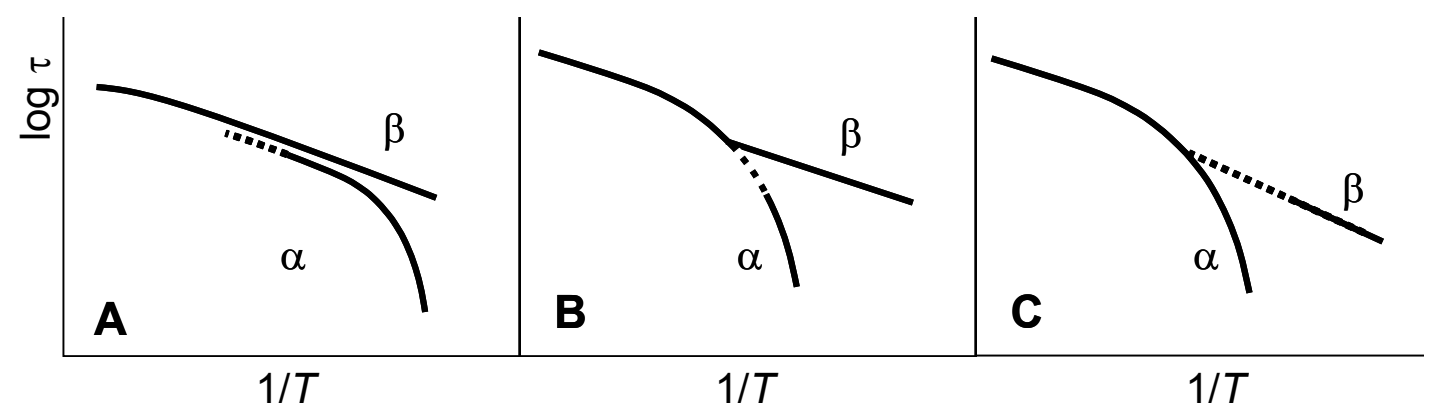

Fig. 9. Three scenarios for the splitting region of the poly(n-alkyl methacrylate)s in the Arrhenius diagram (adapted from ref. [62]); see text

In this type of polymers, the side group is attached to the main chain through a carbon atom, resulting in a loss of rotational freedom relatively to polymers where the bond is through an oxygen atom (e.g., poly(vinyl acetate)). Moreover, the methyl group directly attached to the carbon of the main chain that carries the polar side group confers an additional stiffness to the main chain, which further hinders the rotational mobility of the ester side group. This particular molecular architecture determines the location of both the $\alpha$ and $\beta$ processes and of the region where they merge. The superposition of $\alpha$ and $\beta$ relaxations at temperatures higher than $T_{\mathrm{g}}$ is sensitive to molecular details, following different splitting scenarios. The study of the $\alpha \beta$ coupling in poly(n-alkyl methacrylate)s has the advantage of both secondary and main processes being associated with high dielectric strength values, contrary to a variety of other materials where the $\beta$ relaxation is much less intense, as illustrate before for poly(vinyl acetate). An additional advantage is that the location of the $\alpha \beta$ splitting region in these polymers is accessible by both dielectric and mechanical spectroscopies, although it implies to cover a broad range of both frequencies and temperatures since it deviates to lower temperatures and frequencies as the size of the alkyl side group increases. The resolution of both $\alpha$ and $\beta$ processes in higher poly(n-alkyl methacrylate)s can be achieved by measurements performed at very low frequencies or by applying moderate pressure $[66,67]$.

Among the poly(n-alkyl methacrylate)s, the most studied ones are PMMA (poly(methyl methacrylate)) [64,68-72], PEMA (poly(ethyl methacrylate)) [64,66-67,69-74] and PnBMA (poly(n-butyl methacrylate)) [64,69-70,72-74], mainly by DRS and NMR. The first studies by dielectric relaxation spectroscopy of poly(n-alkyl methacrylate)s (revised in ref. [3]) reveal some inconsistencies due to the $\alpha \beta$ coupling. In spite of that, some general features were found, namely the $\beta$ relaxation that in the lower alkyl methacrylates reveals to be an intramolecular process in nature independently of the side group, with an activation energy in the range of $19-23 \mathrm{kcal} / \mathrm{mol}$, and the $a$ process that is strongly determined by the $\alpha$ relaxation. The development of the NMR technique and the increase of the frequency window in DRS brought new insights into the molecular dynamics of these materials. Some contributions will be analysed 
next. The dielectric loss spectra for PMMA, the lowest member of the series, reveals an intense $\beta$ process whose intensity increases with the temperature increase, coupling with the $\alpha$ relaxation at temperatures around $100-120^{\circ} \mathrm{C}$ and evolving for a single a process at around $150^{\circ} \mathrm{C}[68,75,76]$. Fig. 10 presents the coupling of $\alpha$ and $\beta$ processes for PMMA.

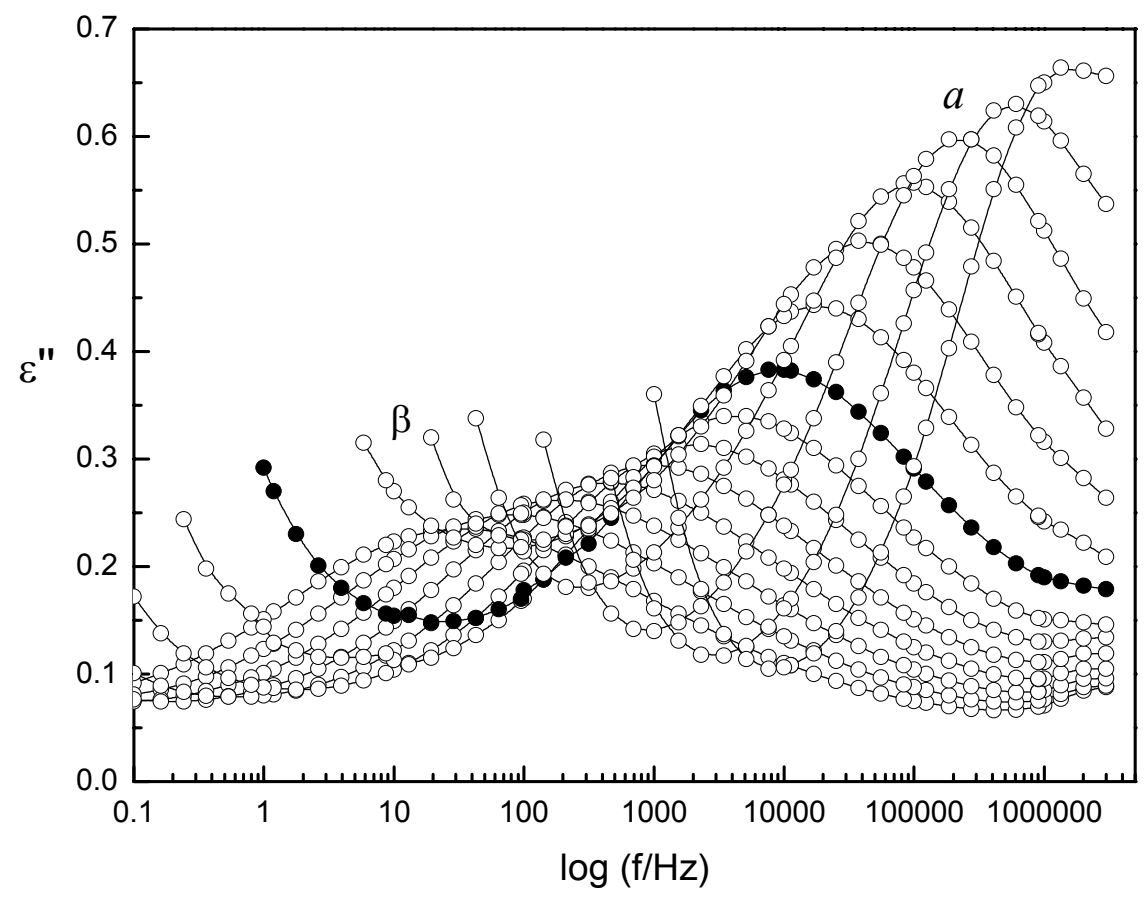

Fig. 10. Dielectric loss curves for PMMA in the temperature region from 30 up to $170^{\circ} \mathrm{C}$ in steps of $10^{\circ} \mathrm{C}$; the full symbols indicate the onset of the $\alpha$ coupling at $110^{\circ} \mathrm{C}$

PMMA and PEMA studied by NMR $[71,72]$ reveal that the $\beta$ process at temperatures below but near $T_{\mathrm{g}}$ is not strictly local but rather consists of $\pi$ flips of the carboxyl group accompanied by restricted rearrangements of the main chain. In PMMA the side group reorients rapidly and couples with the time scale of the $\alpha$ process. Owing to this, the mobility of the main chain is particularly high at temperatures slightly above $T_{\mathrm{g}}$ reorienting in a relatively isotropic mode. To the contrary, in PEMA, the mobility of the side group with a larger dimension induces anisotropy in the movements of the main chain and slows its dynamics by about two orders of magnitude, relatively to PMMA.

The coordinated mobility in the $\beta$ process is in the origin of the designation adopted by Garwe et al. [64], as 'locally coordinative' process. In PEMA the $\beta$ process seems to act as a precursor of the cooperativity of the $\alpha$ process - scenario A in Fig. 9 - with onset at around $110^{\circ} \mathrm{C}$, giving rise to a splitting profile different from PnBMA and higher poly(n-alkyl methacrylate)s. PMMA, like PEMA, presents an activation plot in the splitting region compatible with the A scenario as illustrated by the data of Sy and Mijovic [75].

In the higher poly(n-alkyl methacrylate)s with $\mathrm{R}=\mathrm{nBMA}$ and superior the main chain contributes to both $\alpha$ and $\beta$ processes, performing at the same time local and cooperative dynamics; the side group only contributes to the $\beta$ relaxation that preserves some independent dynamics relatively to the main chain [1]. The increase of the side 
group length shifts the splitting region to lower temperatures and frequencies and thus the onset of the $\alpha$ process in PnBMA is around $50^{\circ} \mathrm{C}$ [74]. As the temperature decreases the a process bifurcates in both $\alpha$ and $\beta$ processes in a parallel mode in the activation plot, with the $\alpha$ process located one decade below the straight line of the $\beta$ process - scenario B in Fig. 9. PnHMA (poly(n-hexyl methacrylate)) presents a similar scenario $[1,77]$ with the dielectric $\alpha$ onset at $9 \pm 10^{\circ} \mathrm{C}$ [77]. Also PnOMA (poly(n-octyl methacrylate)), presents an $\alpha \beta$ splitting B scenario, leading us to think that this is the characteristic profile for the higher poly(n-alkyl methacrylate)s. This $B$ scenario is highly sensitive to molecular details, e.g., in PnBMA/polystyrene copolymers the $B$ scenario translates to $C$ with increasing amount of polystyrene (see Fig. 9) [64]. (The herein designated $B$ scenario is the A scenario in ref. [64].)

\section{Behaviour above the $\alpha \beta$ splitting}

It is usual to estimate the properties in the region above the $\alpha \beta$ splitting by extrapolating the glass transition behaviour by applying the WLF equation (Eq. (10)), i.e., the validity of the time-temperature superposition is assumed. However, there are some studies that do not legitimate this assumption, namely studies by dynamic neutron scattering [78], where it is shown by an unequivocal mode, for several polymers, that the dynamics of the a process is dominated by the Rouse modes or $R$ modes of Rouse-Zimm, which are inhibited in higher length scales or inferior frequencies due to entanglements. Therefore, there are different dynamics below and above the $\alpha \beta$ splitting region.

This change in molecular dynamics is enhanced through the analysis of the derivatives $d / d T, d / d(1 / T)$ and $d^{2} / d T^{2}$ of log $\omega_{\max }$ that makes linear the different dependence laws $\tau(T)$, namely the VFTH law, as proposed for glass formers by Stickel, Fischer and Richert [79]. By this way it became possible to solve subtle variations of $\tau(T)$ that are less obvious in the usual representation of $\log \omega_{\max } v s$. $T$, and it was concluded that the temperature dependence of the $\alpha$ relaxation time changes at a particular temperature, $T_{\mathrm{B}}$, which almost coincides with the temperature where the $\alpha \beta$ coupling occurs [79-81]. The change of molecular dynamics, also observable by the change of the $\beta_{\mathrm{Kww}}$ parameter that largely increases with decreasing temperature [81], is interpreted as the onset of a significant cooperativity at temperatures below $T_{\mathrm{B}} \approx T_{\beta}$.

The absence of significant cooperativity above the $\alpha \beta$ splitting has been observed for a variety of polymers through the $\Delta C_{p}$ jump that tends to zero in that region $[82,83]$ (the $\Delta C_{p}$ jump is considered as an indicator of cooperativity in the glass transition). In the concrete, PnOMA studied by heat capacity spectroscopy [65] showed that RouseZimm-like modes are not accompanied by a specific cooperativity and the relaxation processes detected below and above the $\alpha \beta$ splitting are not related by the timetemperature superposition principle. The same technique applied to PnHMA [77] shows an extinction of the a process clearly separated from the onset of the $\alpha$ process and characterises the a process as coordinative (coordinated mobility on a length scale $<1 \mathrm{~nm}$ ) and the $\alpha$ process as a cooperative process whose cooperativity increases with the temperature decrease below the onset, involving length scales greater than $1 \mathrm{~nm}$.

Therefore, the a process that arises at temperatures above the $\alpha \beta$ coalescence is a distinct process and not a simple superposition of $\alpha$ and $\beta$ relaxations, as already argued by Williams $[66,67]$ for PEMA. Thus, different concepts and formalisms are 
necessary to understand on a molecular level the dynamics in the regions below and above the $\alpha \beta$ splitting.

\section{Relaxations in semi-crystalline polymers}

The last sections addressed the molecular mobilities that may occur in disordered polymeric systems. The presence of crystallinity may affect the molecular dynamics in two main ways:

i) A fraction of the amorphous phase may be partially confined within the crystalline lamellae, in a quasi-bidimensional geometry with a thickness of, typically, between 2 and $10 \mathrm{~nm}$. Therefore, the chains will have a constrained mobility and we may expect serious changes in the glass transition dynamics. The Adam-Gibbs theory [31] introduced the concept of the cooperatively rearranging region (CRR) that defines the smallest region around a relaxing unity that may undergo a transition to a new configuration without requiring configurational changes outside its boundaries. Such regions around $T_{\mathrm{g}}$ have length scales of $1.0-3.5 \mathrm{~nm}$ [84]. So, if chains in the amorphous regions are constrained within geometries below such sizes, they are incapable to relax with the same characteristic times as the unconstrained chains, and will exhibit higher $T_{\mathrm{g}}$ values. As it will be seen, the confinements imposed by the crystalline lamellae are enough to affect the segmental dynamics in polymers. Local motions that will give rise to sub- $T_{\mathrm{g}}$ relaxations are not affected by the presence of crystallinity. It should be pointed out that the dynamics of glass-forming liquids in confined geometries has been widely studied in the last decade. Usually, the confinement is imposed by nano-pores of controlled dimensions $[85,86]$ or trough the preparation of ultra-thin films [87].

ii) The existence of crystalline structures may introduce new relaxation processes involving motions within the crystallites, or loss processes due to mobility between crystalline regions. These effects are found in some flexible polymers, where at least one new relaxation process, labelled $\alpha_{\mathrm{c}}$, appears above $T_{\mathrm{g}}$.

The two mentioned effects will be analysed separately, and examples will be given.

\subsection{Influence of crystallinity on the glass transition dynamics}

In a semi-crystalline polymer the conformational mobility of the chains of the amorphous phase is significantly limited near the crystallites. This means that the glass transition in semi-crystalline polymers depends on the crystalline fraction and also on its microstructure. In particular, the existence of interfacial regions with properties lying between those of crystalline and undisturbed amorphous regions has been revealed by quantitative comparisons of the degree of crystallinity, determined by distinct methods $[88,89]$. Although the existence of the order-disorder transition region at the crystal-amorphous boundaries is well demonstrated, until the recent work of Ivanov and co-workers [90] there was no direct experimental demonstration of the crystal-amorphous interface. This evidence was obtained by transmission electron microscopy (TEM) observation of the compositional variation of stained PET segments in the boundary of an isolated lamellar stack in a binary blend of semicrystalline PET with amorphous poly(ether imide) (PEI) [90].

From a morphological point of view, there is a fraction of non-crystalline material that is not able to exhibit the relaxational characteristics of liquid-like amorphous regions and thus does not contribute, e.g., to the changes in the dielectric permittivity and the 
specific heat capacity that occur at the glass transition. This so-called rigid amorphous phase [91], to be distinguished from crystals and from a mobile (undisturbed) amorphous phase, is probably the result of restrictions of molecular mobility due to the fixation of the polymer chains at the surface of the crystalline lamellae.

PET can be easily obtained either as closely amorphous or as a semi-crystalline material, in a range of crystallinities that can vary from c. $0-50 \%$, as a result of thermal treatments above the glass transition. Therefore, this material is a model system for studying the influence of crystallinity on the glass transition dynamics.

Figs. $4 \mathrm{a}$ and $4 \mathrm{~b}$ correspond, respectively, to a PET sample quenched from a temperature above $T_{\mathrm{g}}$ to a sufficiently low temperature, below $T_{\mathrm{g}}$, and to the same PET kept at $163^{\circ} \mathrm{C}$ for $1 \mathrm{~h}$. From the first thermal treatment a nearly amorphous sample results, whereas with the second treatment a semi-crystalline sample with crystallinity degree $X_{c} \approx 36 \%$ is obtained. It can be seen that the main effects of the strong coupling between the crystalline and amorphous phases are the increase of $T_{\mathrm{g}}$ and the broadening of the glass transition. The glass transition of the amorphous PET covers a temperature interval from 60 to $80^{\circ} \mathrm{C}$ and the calorimetric $T_{\mathrm{g}}$ is $71^{\circ} \mathrm{C}$ while the glass transition of the semi-crystalline PET covers the temperature interval between 70 and $110^{\circ} \mathrm{C}$ and $T_{\mathrm{g}}$ is $\approx 81^{\circ} \mathrm{C}$. Of course, the increase of the specific heat capacity associated to the glass transition, $\Delta C_{p}\left(T_{g}\right)$, decreases as the crystallinity degree increases. In the example of Fig. $5, \Delta C_{p}\left(T_{\mathrm{g}}\right)=0.33 \mathrm{~J} /(\mathrm{g} \mathrm{K})$ for the amorphous PET and $\Delta C_{p}\left(T_{\mathrm{g}}\right)=0.13 \mathrm{~J} /(\mathrm{g} \mathrm{K})$ for the semi-crystalline PET.

The behaviour of low-crystallinity PET is also an excellent example of the influence of crystallinity on the glass transition dynamics. In this case, two distinct amorphous phases have been detected by several techniques. In particular, DSC has been especially useful to study the presence of these two phases due to the possibility of analysing the effects of physical ageing on the two amorphous phases, after subjecting the samples to distinct thermal treatments [36].

Fig. 11 corresponds to the same PET as Fig. 5, but subjected to a thermal treatment (described in Fig. 11) from which a low-crystallinity sample results. Two amorphous phases are revealed by the presence of two ageing peaks. One phase has a $T_{\mathrm{g}}$ very similar to that of amorphous PET (Fig. 5a) and the other phase has a higher $T_{\mathrm{g}}$, close to that of the higher-crystallinity PET (Fig. 5b). The origin of the two amorphous phases in PET should be consistent with its spherulitic morphology. In fact, the growth of spherulites and of the lamellar structure is not completed when amorphous PET is crystallised at relatively low temperatures for short periods of time. In these conditions it is expected that the $\alpha$ relaxation has two contributions: one from the amorphous parts far from the crystallites (phase I) and another from the amorphous parts strongly confined between the crystalline lamellae (phase II). It is assumed that in phase I the conformational rearrangements occur in a similar way as in the fully amorphous polymer and in phase II the polymer chains have a limited mobility. As crystallisation proceeds or the crystalline fraction changes, the relative contribution of these two fractions to the $\alpha$ relaxation behaviour also changes.

For PET with higher crystallinity (as the sample of Fig. 5b) the rigid amorphous phase is formed by chains with even higher restrictions of molecular mobility than the ones of phase II, probably due to its fixation at the surface of the crystalline lamellae [91]. It is believed that the glass transition of the mobile amorphous phase is affected by the rigid amorphous phase [91], namely it broadens this relaxation to higher temperatures. A recent work showed that the correlation length for the cooperative motion of 
polymer chains in the amorphous phase increases with increasing thickness of the rigid amorphous phase [92].

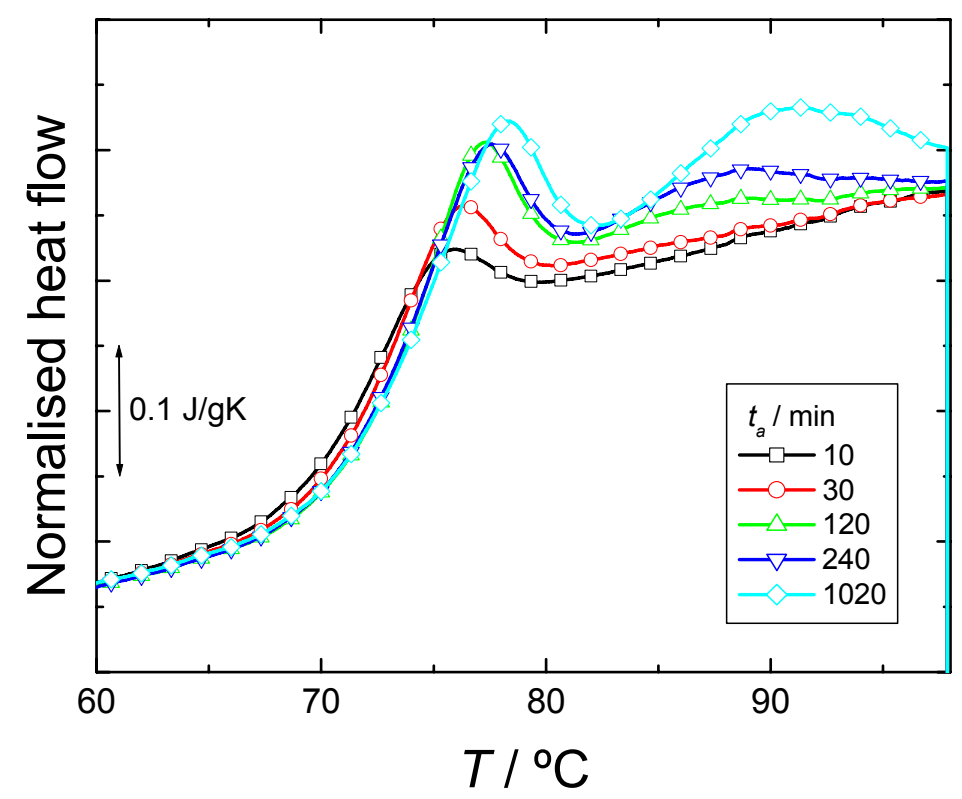

Fig. 11. Temperature dependence of the normalised heat flow of low-crystallinity PET, previously subjected to distinct annealing periods (in the graphics) at $58^{\circ} \mathrm{C}$. Low-crystallinity PET was prepared by heating an amorphous sample from $30^{\circ} \mathrm{C}$ to $131^{\circ} \mathrm{C}$ at $20^{\circ} \mathrm{C} / \mathrm{min}$. At this temperature, the DSC scan reached $19 \%$ of the total crystallisation peak area. Data taken from ref. [36]

The effect of crystallinity on the glass transition is also reflected on the characteristic time distribution. Looking again at the example of Fig. 5, the $\beta_{\mathrm{Kww}}$ parameter obtained for the amorphous sample is significantly higher $\left(\beta_{\mathrm{KWw}}=0.41\right)$ when compared with the value found for the semi-crystalline sample $\left(\beta_{\mathrm{Kww}}=0.22\right)$, indicating that the distribution of relaxation times is broader for the latter [36]. The close interaction with the crystallites makes it easier to find amorphous rearranging regions with distinct mobility. The same variation of the $\beta_{\mathrm{kww}}$ parameter with crystallinity was found by DRS [93].

\section{2. $\alpha_{c}$ Relaxation: the polyethylene example}

Polymers with a flexible chain, such as polyethylene (PE), polypropylene, polytetrafluoroetylene or poly(methylene oxide), exhibit relaxation processes directly related to the presence of the crystalline fraction. For the case of PE, by far the most important system in this context, such processes may be dielectrically active, provided that it is decorated with a few $\mathrm{C}-\mathrm{Cl}$ or $\mathrm{C}=\mathrm{O}$ dipoles, by chlorination or oxidation [94]. Even simple melt-based processing may induce some oxidation in PE.

The $\alpha_{c}$ relaxation appears between $T_{\mathrm{g}}$ and the melting temperature. For PE it is complex and comprises at least two relaxation mechanisms, named $\alpha$ land $\alpha$ ll [95] or $\alpha$ and $\alpha^{\prime}$ [96], that cover the temperature range between 30 and $120^{\circ} \mathrm{C}$. The study of this general process is important as it is related to relevant materials' properties such as creep, annealing, crystallisation, extrudability and drawability [96]. One should distinguish the $\alpha_{c}$ relaxation from the $\alpha$ relaxation found in amorphous systems, 
related to cooperative motions that give rises to the glass transition. In such semicrystalline systems the glass transition dynamics is assigned to the $\beta$ relaxation. This relaxation is well detected in low-density $\mathrm{PE}$ at $c .0^{\circ} \mathrm{C}$ (for frequencies around $1 \mathrm{~Hz}$ ) but is very weak in high-density PE, due to the low content of amorphous phase.

There are many studies that searched for a relationship between the $\alpha_{c}$ relaxation and its origin on the molecular level. General information in this context may be found in many books or review papers (e.g., refs. [3,98-100]), and here only a brief review of the subject will be made. DRS and NMR results demonstrated that the $\alpha_{c}$ process is associated with rotational motions within the crystalline lamellae. A $180^{\circ}$ flip in a molecular segment occurs at a given place and propagates in screw-like motions throughout the crystal. This will result in an effective translation of a carbon atom of the chain (i.e., half of a unit cell). This mechanism involves displacements within the chain along states that are energetically equivalent, and thus it could not originate a mechanical response. However, there is a mechanical register of the $\alpha_{c}$ relaxation that results from an additional shear of the amorphous regions. For this, there is the need for chain transport through the crystallites, that occurs by a long-distance solidstate diffusion process, with an activation energy, for the case of linear PE, of 105 $\mathrm{kJ} / \mathrm{mol}$ [101]. Such a value is consistent with the activation energies for the $\alpha_{c}$ relaxation, which may vary between 90 and $300 \mathrm{~kJ} / \mathrm{mol}$. Therefore, in the $\alpha_{c}$ process one relaxation mode within the crystals and another one in the amorphous regions are combined. Note, however, that the $\alpha_{c}$ relaxation observed by mechanical spectroscopy is considerably broader than by dielectric spectroscopy (the mechanical ColeCole width parameter is $\approx 0.4$, while the dielectric one is $0.7-0.8$ [97]), reflecting the variety of environments felt by the chains in the amorphous phase.

A non-solved issue of the $\alpha_{c}$ relaxation is to know if it is a really thermally activated process. The Arrhenius plots are found to be non-linear [102-104] (see also Fig. 12), but this can be attributed to the overlapping of different thermally activated processes.
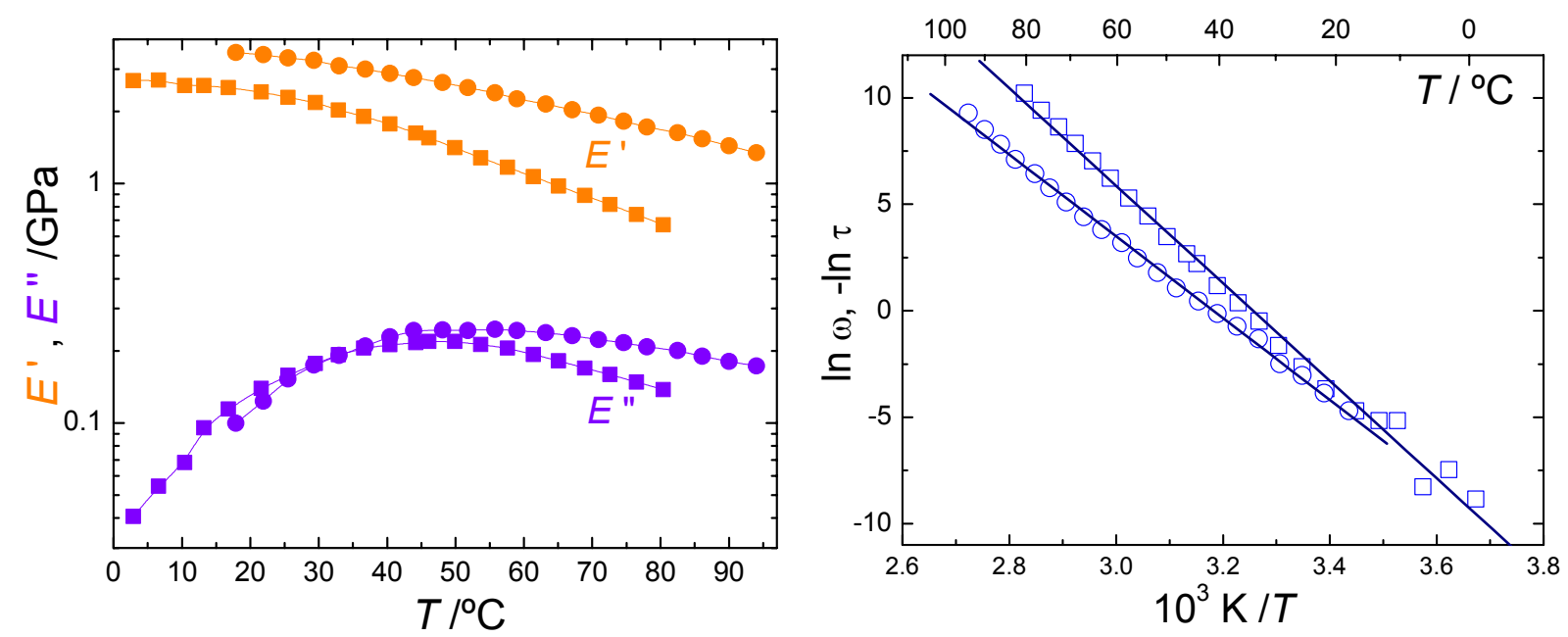

Fig. 12. Left - storage modulus (orange) and loss modulus (violet) in the $\alpha_{c}$ relaxation region of high-density $\mathrm{PE}$, observed at $1 \mathrm{~Hz}$. The squares correspond to a quasiisotropic material, obtained by conventional injection moulding, whereas the circles are for the same material processed in such a way that much more molecular orientation was induced. Right - Arrhenius plot for the two materials. The results were taken from refs. [103,104] 
If one considers that the $\alpha_{c}$ relaxation(s) is (are) thermally activated then conclusions can be drawn about the cooperative nature of the process: is the $\alpha_{c}$ relaxation simple, involving only motion of chains that do not interfere with the neighbouring groups of atoms and molecules, or, on the contrary, do the molecular motions involve, in part, a range of related motions within a significant correlation volume? Results such as the ones shown in Fig. 12 were analysed to extract the activation energies. These values were introduced in an $E_{a} v s$. $T$ diagram, together with a line obtained from the hypothetic case, also at a frequency of $1 \mathrm{~Hz}$, where the activation entropy is zero. A process with negligible activation entropy is considered as a simple, non-cooperative process, whereas appreciable activation entropies correspond to cooperative processes $[105,106]$. This method may be also used in lowmolecular weight materials [107]. Considerable deviations were found between the experimental activation energies and the one predicted for the zero entropy case [104], indicating that the $\alpha_{c}$ relaxation is substantially cooperative. This was attributed to the involvement of the amorphous region in this process, which will increase the complexity of the motions involved. It should be added that a similar analysis allowed demonstrating that the $\alpha_{c}$ relaxation, as seen by dielectric or NMR tests, is also cooperative, although in less extent.

The above results demonstrate the intrinsic difference between the dielectric and mechanical responses of the $\alpha_{c}$ relaxation. However, it must be considered that the activation energies measured by the two techniques are similar, as well as the effect of the thickness of the lamellae on the position of the relaxation in the temperature axis.

\section{Molecular mobility in liquid crystalline polymers}

In the previous sections the molecular mobility was analysed in both amorphous and semi-crystalline systems. Crystalline structure is characterised by a defined arrangement of the molecules in the space, whereas in the amorphous or liquid state no long-range order exists, neither in a positional nor in an orientational point of view. Liquid crystalline polymers (LCPs) may exhibit a combination of properties that appear in crystalline substances (e.g., optical anisotropy) and in amorphous polymers (e.g., flow capability and thus the possibility to be processed by conventional techniques) $[108,109]$. In thermotropic LCPs one or more liquid crystalline phases may be detected along the temperature axis, where the relevant molecular entities (mesogenic groups), with a highly anisotropic geometry and rigid structure, shows some orientational ordering capability. The existence of mesogenic groups enables the appearance of new phases, and one expects that the dynamics in such systems will further complicate. DRS has been the technique most used to characterise the molecular mobility in LCPs, due to the huge frequency range that may be acceded and because of the polar nature of these polymers (especially in the mesogenic groups) [110].

Like in conventional amorphous polymers, LCPs present local relaxations $(\beta, \gamma, \ldots)$. For non-crystallisable systems (e.g., side-chain LCPs, where the mesogenic groups are attached to the main chain through a flexible spacer, usually a short alkyl chain), one may find, besides the $\alpha$ relaxation, a new relaxation at higher temperatures (or at lower frequencies), labelled $\delta$. As an example, Fig. 13 shows the loss factor obtained by both mechanical and dielectric spectroscopies, at $1 \mathrm{~Hz}$, of a side-chain LCP, near and above $T_{\mathrm{g}}$. Both $\alpha$ and $\delta$ relaxations may be detected. 


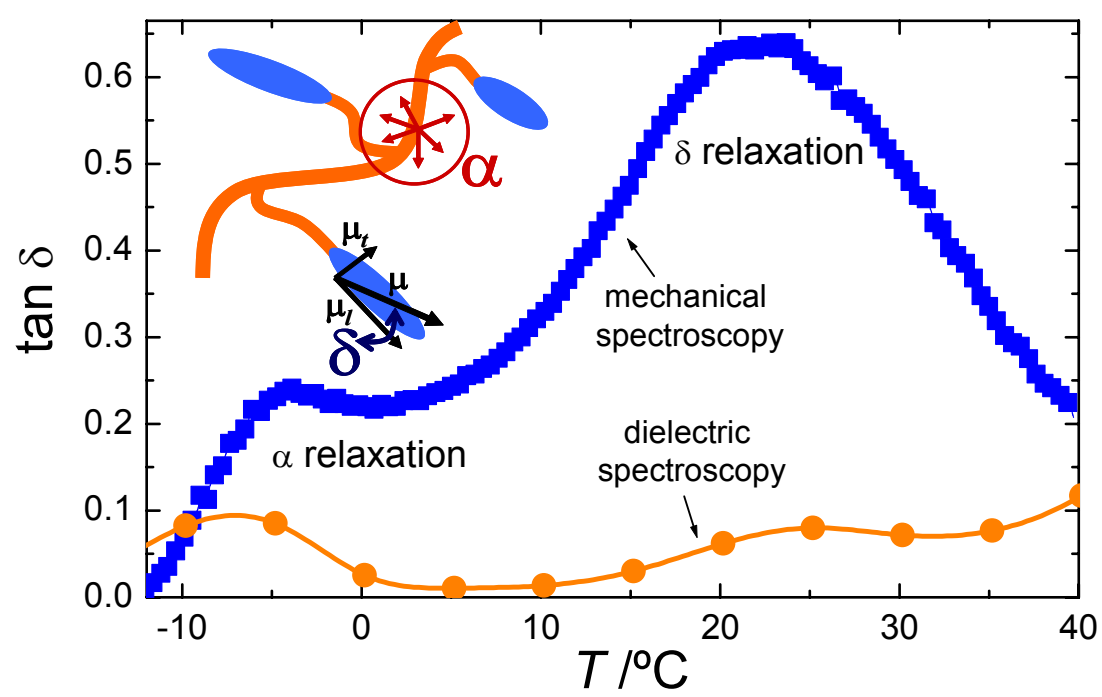

Fig. 13. Loss factor of a side-chain LCP (polysiloxane) measured at $1 \mathrm{~Hz}$ using both dielectric relaxation spectroscopy and dynamic mechanical analysis (compression mode). The results were extracted from ref. [111]. In the inset a scheme of a sidechain LCP structure is shown with the main chain, the spacers and the mesogenic groups (elliptic shapes). Molecular origins of $\alpha$ and $\delta$ relaxations are also represented

The attribution of these processes on the molecular level is still controversial, mainly for the $\alpha$ relaxation. Some authors claim that the $\alpha$ relaxation is assigned to the cooperative motions associated with the glass transition (see scheme in Fig. 13). However, there are others that attribute this process to special motions, involving mainly the transverse component of the mesogenic dipolar moment (see a deeper discussion on this subject in, e.g., ref. [111]).

The $\delta$ relaxation is mainly associated with the mobility modes of the longitudinal component of the dipolar moment of the mesogenic groups (see inset scheme in Fig. 13). It is not yet clear if such motions are linked to the dynamics of the main chain. In fact, dynamics tends to adopt a VFTH behaviour when the temperature approaches $T_{\mathrm{g}}$, and sudden changes in the mechanism occur in the transition between the liquid crystalline phase and the isotropic liquid. It is interesting to notice that, although being associated to special motions in the dipolar groups, the $\delta$ relaxation is also mechanically active and characterised by a narrow distribution of relaxation times (see Fig. 13). This process is not active by DSC, because the changes in entropy are not sufficient to be traduced into a significant change of heat capacity [111]. This is related to the fact that the occurrence of the $\delta$ relaxation does not increase considerably the number of microstates $\Omega$ that are intimately related to the system's entropy by the Boltzmann formula $S=k \ln \Omega$. Just for comparison, during the glass transition there is an exponential increase of the number of configurational states with increasing temperature. In this case a change in the heat capacity, $C_{p}=T \mathrm{~d} S / \mathrm{d} T=k T$ $\mathrm{d}(\ln \Omega) / \mathrm{d} T$, is perfectly noticeable. In semi-crystalline polymers, the same absence of a DSC signal is found for the $\alpha_{c}$ relaxation. It may be explained using the same arguments used for the $\delta$ relaxation: no extensive changes in $\Omega$ are observed with increasing temperature due to the screw-like motions occurring within the crystalline structure. Other similarities can be found between $\alpha_{c}$ and $\delta$ relaxations, such as the similarities in the activation energies, the narrow distribution of characteristic times and the location above $T_{\mathrm{g}}$ [112]. Moreover, the $\delta$ relaxation also exhibits a certain degree of cooperativity, despite involving only fluctuations on the dipolar moment of 
the mesogenic groups. Such cooperativity was found in a large series of LCPs, with very different chemical structures, being higher than the one found in local motions below $T_{\mathrm{g}}$ (e.g., $\beta$ relaxations) and similar to the cooperativity found for the $\alpha_{\mathrm{c}}$ relaxation in PE [112]. In such analysis the same concept of activation entropy was used as previously referred to. This means that the conformational motions within a mesogenic group associated with the $\delta$ relaxation oblige neighbouring groups to move throughout a given correlation volume. Therefore, the existence of large amplitude motions along the principal molecular axis of the mesogenic groups may take place during this process.

Several arguments have been provided that strengthen the attribution of the $\alpha$ relaxation to the glass transition dynamics, as it is found in conventional amorphous polymers: i) the temperature dependence of the characteristic times follow VFTH dynamics (see Fig. 14); ii) the distribution of relaxation times is also similar to the one observed in amorphous systems; iii) the temperature of maximum $\varepsilon$ " at low frequencies (around $10^{-2} \mathrm{~Hz}$ ) is similar to the calorimetric glass transition [113,114]; iv) the features of glass transition dynamics as studied by structural relaxation using DSC are similar to the dielectric $\alpha$ relaxation [115]; v) the temperature-modulated DSC technique allowed to show that the glass transition of a LCP follows the same kinetics as the dielectric $\alpha$ relaxation [116].

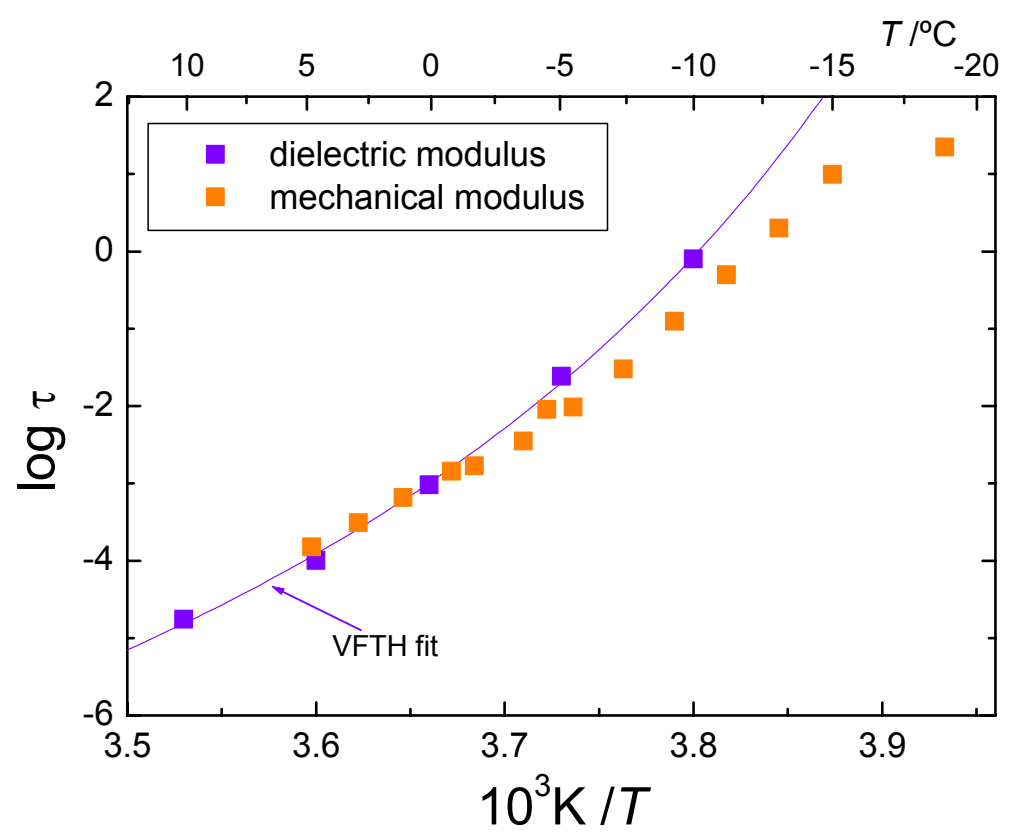

Fig. 14. Arrhenius plot for the $\alpha$ relaxation of the same LCP assigned to the data in Fig. 10, obtained from dielectric (violet) and mechanical (orange) spectroscopies. The solid line is the VFTH fitting of the dielectric data. Data taken from ref. [111]

However, even if one assigns the $\alpha$ relaxation found in LCPs to the glass transition, its dynamics is still highly dependent on the existence of the bulky mesogenic groups, as well on the spacers. It was suggested [111] that the $\alpha$ relaxation would be assigned to conformational mobility in the main chain, but these motions would occur with, or after, the onset of the fastest motions in the mesogenic groups, that would include the fluctuations of the transverse component of their dipolar moment. It was found that the KWW exponent, $\beta_{\mathrm{KWW}}$, of a LCP is lower than the one of the corre- 
sponding polymer without side chain [112]. This would correspond to an enlargement of the characteristic time spectrum due to the existence of local concentration fluctuations of the spacer chains.

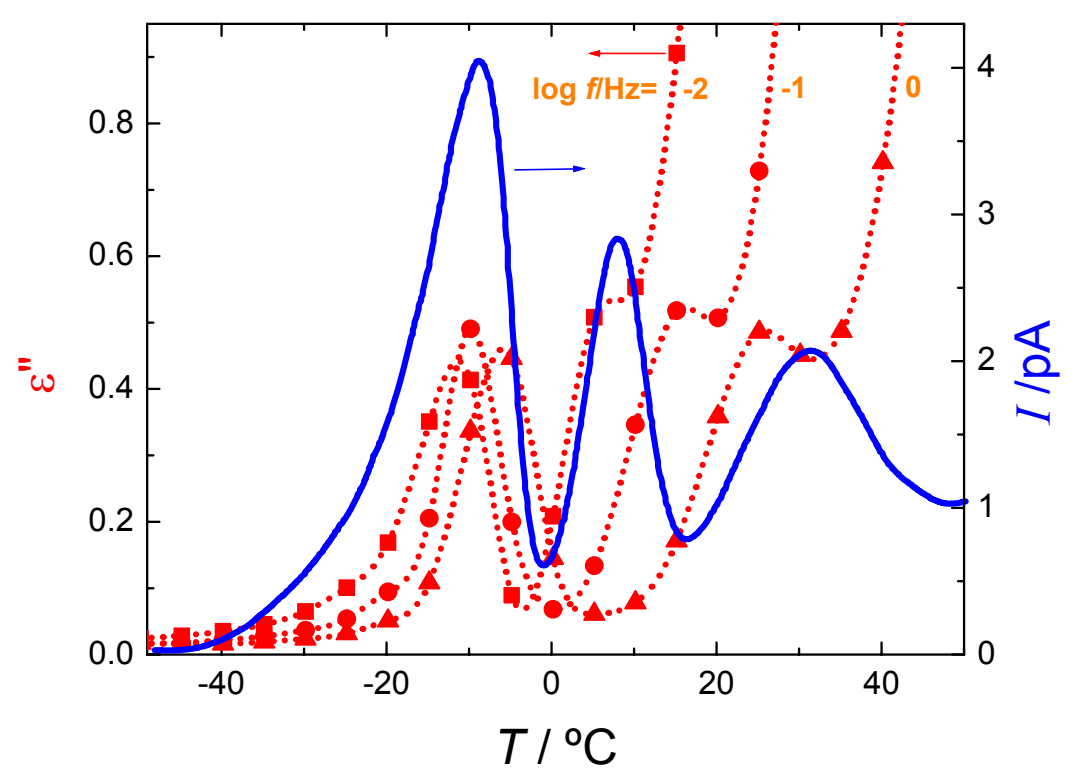

Fig. 15. Comparison between the dielectric responses in the temperature domain of the same LCP as in Figs. 13 and 14 obtained by (i) dielectric spectroscopy (in red), at different frequencies, and (ii) by thermally stimulated depolarisation currents (in blue). The data were taken from ref. [112]

Transient tests may also be useful in the study of molecular mobility in polymers, and in particular LCPS. The thermally stimulated depolarisation currents technique (TSDC) is based on the capability of a sample to polarise at high temperature, under the effect of a static electric field, and then keep a permanent polarisation at low temperature without the effect of the field (at low temperatures the characteristic times of the relaxation processes under study will be high enough to prevent the corresponding conformational motions to occur). During heating, the dipolar polarisation may be released in different temperature intervals, corresponding to the onset of specific motion modes, when the time scales of these processes are equivalent to the time scale of the experiment (typically $100-1000 \mathrm{~s}$, depending on the scanning rate). Experimentally, the depolarisation processes are monitored by the reading of the electric current that is produced in the sample. Fig. 15 shows the example of a global TSDC experiment of a side-chain LCP - the same material as studied in Figs. 13 and 14 . Three main relaxation processes may be detected in this temperature window. In the same temperature range, the dielectric loss at different frequencies is also included. One may find, from these dynamic experiments, the $\alpha$ relaxation at lower temperatures and, for higher temperatures, the $\delta$ relaxation. At higher temperatures the conductivity tail is well visible, especially for lower frequencies. Fig. 14 proves that, if one extrapolates the dielectric spectroscopy results to low frequencies (TSDC experiments have equivalent frequencies of $10^{-3}-10^{-2} \mathrm{~Hz}$ [117]) the $\alpha$ relaxation will superimpose to the low-temperature relaxation (at $\approx-10^{\circ} \mathrm{C}$ ) measured by TSDC, which will thus be assigned to the glass transition dynamics. Moreover, the peak corresponding to the $\delta$ relaxation would correspond to the process at $\approx 9^{\circ} \mathrm{C}$ found by TSDC. Finally the TSDC peak at $\approx 30^{\circ} \mathrm{C}$ would correspond to a non-dipolar 
process (motions of spatial charges), being consistent with the conductivity tail found by DRS.

LCPs exhibit ferroelectric properties in chiral tilted smectic phases (e.g., $S_{\mathrm{c}}{ }^{*}$ phase). Two collective loss processes have been reported in such cases, from frequencies below $1 \mathrm{MHz}$ : the Goldstone and the 'soft' mode. The former is assigned to thermal fluctuations or to modulations induced by the field, respectively, of the phase angle of the helical superstructure, which is connected to the different polarisation vector of the smectic layers. The soft mode is attributed to amplitude fluctuations of the ferroelectric helix. At higher frequencies $(1 \mathrm{MHz}-10 \mathrm{GHz})$ the $\beta$ relaxation is detected, being assigned to fluctuations in the mesogenic groups along their long molecular axis. Further information about this subject may be found elsewhere (e.g., ref. [118] and refs. cited therein).

[1] Ovidiu, P.; Beiner, M.; Reichert, D.; Macromolecules 2003, 36, 3992.

[2] Richert, R.; Blumen, A.; editors; "Disorder Effects on Relaxational Processes", Springer-Verlag, Berlin 1994.

[3] McCrum, N. G.; Read, B. E.; Williams. G.; "Anelastic and Dielectric effects in Polymeric Solids", Wiley, New York 1967 (reprinted by Dover, New York 1991).

Schönhals, A; Kremer, F.; editors; "Broad Band Dielectric Spectroscopy", SpringerVerlag, Berlin 2003.

[4] Strobl, G.; “The Physics of Polymers”, Springer, Berlin, Heidelberg 1996.

[5] Hunt, B. J.; James, M. I.; "Polymer Characterisation", Blackie Academic \& Professional, Suffolk 1993.

[6] Schönhals. A.; in "Dielectric Spectroscopy of Polymeric Materials", Runt, J. P.; Fitzgerald, J. J.; editors; American Chemical Society, Washington 1997.

[7] Johari, G. P.; Goldstein. M. J.; J. Chem. Phys. 1970, 53, 2372.

[8] Ngai, K. L.; Phys. Rev. E 1998, 57, 7346.

[9] Ngai, K. L.; Capaccioli, S.; Phys. Rev. E 2004, 69, 31501.

[10] Ngai, K. L.; Paluch, M.; J. Chem. Phys. 2004, 120, 857.

[11] Schneider, U.; Lunkenheimer, P.; Brand, R.; Loidl, A.; Phys. Rev. E 1999, 59, 6924.

[12] Eyring, H.; J. Chem. Phys. 1936, 4, 183.

[13] Einfeldt, J.; Maißner, D.; Kwasniewski, A.; Prog. Polym. Sci. 2001, 26, 1419.

[14] Viciosa, M.; Dionísio, M.; Silva, R. M.; Reis, R. L.; Mano, J. F.; Biomacromolecules, in press.

[15] Diaz-Calleja, R.; Saiz, E.; Riande, E.; Gargallo, L.; Radic. D.; Macromolecules 1993, 26, 3795.

[16] Dubault, A.; Bokobza, L.; Gandin, E.; Halary, J. L.; Polym. Int. 2003, 52, 1108.

[17] Arrese-Igor, S.; Arbe, A.; Alegría, A.; Colmenero, J.; Frick, B.; Appl. Phys. A 2002, 74, S454.

[18] Spiess, H. W.; Colloid. Polym. Sci. 1983, 261, 193.

[19] Inglefield, P. I. T.; Amici, R. M.; O'Gara, J. F.; Hung, C. C.; Jones, A. A.; Macromolecules 1983, 16, 1552. 
[20] Hutchinson, J. M.; Prog. Polym. Sci. 1995, 20, 703.

[21] Hodge, I. M.; J. Non-Crystalline Solids 1994, 169, 211.

[22] McKenna, G. B.; J. Res. Nat. Inst. Stand. Technol. 1994, 99, 169.

[23] Kovacs, A. J.; Fortschr. Hochpolym. Forsch. 1963, 3, 394.

[24] Perez, J.; Cavaillé, J. Y.; Díaz-Calleja, R.; Gómez-Ribelles, J. L.; Pradas, M. M.; Greus, A. R.; Makromol. Chem. 1991, 192, 2141.

[25] Sasabe, H.; Moynihan, C. T.; J. Polym. Sci., Polym. Phys. Ed. 1978, 16, 1447.

[26] Narayanaswamy, O. S.; J. Am. Ceram. Soc. 1971, 54, 491.

[27] Williams, G.; Watts, D. C.; Trans. Faraday Soc. 1970, 66, 80. Williams, G.; Watts, D. C.; Dev, S. B.; North, A. M.; Trans. Faraday Soc. 1971, 67, 1323.

[28] Moynihan, C. T.; Macedo, P. B.; Montrose, C. J.; Gupta, P. K.; DeBolt, M. A.; Dill, J. F.; Dom, B. E.; Drake, P. W.; Esteal, A. J.; Elterman, P. B.; Moeller, R. P.; Sasabe, H.; Ann. N.Y. Acad. Sci. 1976, 279, 15.

[29] Scherer, G. W.; J. Am. Ceram. Soc. 1984, 67, $504 . \quad$ Hodge, I. M.; Macromolecules 1987, 20, 2897.

[30] Tool, A. Q.; J. Am. Ceram. Soc. 1946, 29, 240.

[31] Adams, G.; Gibbs, J. H.; J. Chem. Phys. 1965, 43, 139.

[32] Gibbs, J. H.; DiMarzio, E. A.; J. Chem. Phys. 1958, 28, 373.

[33] Gomez Ribelles, J. L.; Monleon Pradas, M.; Macromolecules 1995, 28, 5867.

[34] Meseguer Dueñas, J. M; Vidaurre Garayo, A.; Romero Colomer, F.; Más Estellés, J.; Gómez Ribelles, J. L.; Monleón Pradas, M.; J. Polym. Sci., Polym. Phys. Ed. 1997, 35, 2201.

[35] Brunacci, A.; Cowie, J. M. G.; Ferguson, R.; Gómez Ribelles, J. L.; Vidaurre Garayo, A.; Macromolecules 1996, 29, 7976.

[36] Alves, N. M.; Mano, J. F.; Balaguer, E.; Meseguer Dueñas, J. M.; Gómez Ribelles, J. L.; Polymer 2002, 43, 4111.

[37] Alves, N. M.; Mano, J. F.; Gómez Ribelles, J. L.; Macromol. Symp. 1999, 148, 437.

[38] Alves, N. M.; Mano, J. F.; Gómez Ribelles, J. L.; J. Therm. Analys. Calorim. 2002, 70, 633 .

[39] Alves, N. M.; Mano, J. F.; Gómez Ribelles, J. L.; Polymer 2002, 43, 3627.

[40] Anderson, P. W.; Science 1995, 267, 1615.

[41] Angel, C. A.; Science 1995, 267, 1924.

[42] Williams, G.; in "Keynote Lectures in Selected Topics of Polymer Science", Riande, E., editor; CSIC, Madrid 1995, chap. I.

[43] Inoue, T.; Cicerone, T. M.; Ediger, M. D.; Macromolecules 1995, 28, 3425.

[44] Vogel, H.; Phys. Z. 1921, 22, 645.

[45] Fulcher, G. S.; J. Am. Chem. Soc. 1925, 8, 339.

[46] Tammann, G.; Hesse, W.; Z. Anorg. Allg. Chem. 1926, 156, 245.

[47] Ferry, J. D.; "Viscoelastic Properties of Polymers", Wiley, New York 1970.

[48] Doolitle, A. K.; J. Appl. Phys. 1951, 22, 1471. 
[49] Dionísio, M.; Moura Ramos, J. J.; Williams, G.; Polymer 1993, 34, 4105.

[50] Koizumi, N.; Kita, Y.; Bull. Inst. Chem. Res., Kyoto Univ. 1978, 56, 300.

[51] Havriliak, S.; Negami, S.; Polymer 1967, 8, 161.

[52] Havriliak Jr., S.; Havriliak, S.; "Dielectric and Mechanical Relaxation in Materials", Hanser Publ., Munich 1997.

[53] Alvarez, F.; Alegría, A.; Colmenero, J.; Phys. Rev. B 1991, 44, 7306.

[54] Cole, R. H.; Cole, K. S.; J. Chem. Phys. 1941, 9, 341.

[55] Davidson, D. W.; Cole, R. H.; J. Chem. Phys. 1950, 18, 1417.

[56] Alvarez, F.; Alegría, A.; Colmenero, J.; Phys. Rev. B 1993, 47, 125.

[57] Alegría, A.; Guerrica-Echevarría, E.; Goitiandía, L.; Telleria, I.; Colmenero, J.; Macromolecules 1995, 28, 1516.

[58] Williams, G.; Adv. Polym. Sci. 1979, 33, 60.

[59] Williams, G.; in "Chem. Soc. Spec. Period. Reports, Dielectric and related Molecular Processes", Davies, M., editor; 1975, vol. 2, p. 151.

[60] Williams, G.; Adv. Polym. Sci. 1979, 33, 60.

[61] Bengtzelius, U.; Götze, W.; Sjölander, A.; J. Phys. C 1984, 17, 5915.

Leutheuser, E.; Phys. Rev. A 1984, 29, 2765. Götze, W.; Z. Phys. B 1985, 60, 195.

[62] Götze, W.; Sjögren, L.; Rep. Prog. Phys. 1992, 55, 241.

[63] Götze, W.; Sjögren, L.; Z. Phys. B 1987, 65, 415.

[64] Garwe, F.; Schonhals, A.; Lockwenz, H.; Beiner, M.; Schrotter, K.; Donth, E.; Macromolecules 1996, 29, 247.

[65] Beiner, M.; Korus, J.; Donth, E.; Macromolecules 1997, 30, 8420.

[66] Williams, G.; Trans. Faraday Soc. 1966, 62, 2091.

[67] Williams, G.; Edwards, D. A.; Trans. Faraday Soc. 1966, 62, 1329.

[68] Dionísio, M.; Moura Ramos, J. J.; Williams, G.; Polym. Int. 1993, 32, 145.

[69] Tetsutani, T.; Kakizaki, M.; Hideshima, T.; Polym. J. 1982, 14, 305.

[70] Hideshima, T.; Tetsutani, T.; Kakizaki., M.; Polym. J. 1982, 14, 471.

[71] Kuebler, S. C.; Schaefer, D. J.; Boeffel, C.; Pawelzik, U.; Spiess, H. W.; Macromolecules 1997, 30, 6597.

[72] Kulik, A. S.; Beckham, H. W.; Schmidt-Rohr, K.; Radloff, D.; Pawelzik, U.; Boeffel, C.; Spiess, W.; Macromolecules 1994, 27, 4746.

[73] Dionísio, M.; Moura Ramos, J. J.; Williams, G.; Polymer 1994, 35, 1705.

[74] Schröter, K.; Unger, R.; Reissig, S.; Garwe, F.; Kahle, S.; Beiner, M.; Donth, E.; Macromolecules 1998, 31, 8966.

[75] Wing Sy, J.; Mijovic, J.; Macromolecules 2000, 33, 933.

[76] Dionísio, M.; Fernandes, A.; Mano, J. F.; Correia, N.; Sousa, R.; Macromolecules 2000, 33, 1002.

[77] Beiner, M.; Kahle, S.; Hempel, E.; Schröter, K.; Donth, E.; Macromolecules 1998, 31, 8973.

[78] Richter, D.; Willner, L.; Zirkel, A.; Fargo, B.; Fetters, L. J.; Huang, J. S.; Phys. Rev. Lett. 1993, 71, 4158. 
[79] Stickel, F.; Fischer, E. W.; Richert, R.; J. Chem. Phys. 1996, 104, 2043.

[80] Capaccioli, S.; Casalini, R.; Lucchesi, M.; Lovicu, G.; Prevosto, D.; Pisignano, D.; Romano, G.; Rolla, P. A.; J. Non-Cryst. Solids 2002, 307-310, 238.

[81] Léon, C.; Ngai, K. L.; J. Phys. Chem. B 1999, 103, 4045.

[82] Hempel, E.; Beiner, M.; Renner, T.; Donth, E.; Acta Polym. 1996, 47, 525.

[83] Donth, E.; Kahle, S.; Korus, J.; Beiner, M.; J. Phys. I (France) 1997, 7, 581.

[84] Hempel, E.; Hempel, G.; Hensel, A.; Schick, C.; Donth, E.; J. Chem. Phys. B 2000, 104, 2460.

[85] Pissis, P.; Daoukakidiamanti, D.; Aspekis, L.; Christodoulides, L.; J. Phys. Condensed Matter 1994, 6, 325.

[86] Jackson, C. L.; McKenna, G. B.; J. Non-Cryst. Solids 1991, 131, 221.

[87] Wang, X.; Zhou, W.; Macromolecules 2002, 35, 6747.

[88] Chalmers, J. M.; Everall, N. J.; Trends Anal. Chem. 1996, 15, 18.

[89] Jonas, A. M.; Ivanov, D. A.; Yoon, D. Y.; Macromolecules 1998, 31, 5352.

[90] Ivanov, D. A.; Pop, T.; Yoon, D. Y.; Jonas A. M.; Macromolecules 2002, 35, 9813.

[91] Wunderlich, B.; Prog. Polym. Sci. 2003, 28, 383.

[92] Hong, P.-D.; Chuang, W.-T.; Yeh, W.-J.; Lin, T.-L.; Polymer 2002, 43, 6879.

[93] Ezquerra, T. A.; Baltá-Calleja, F. J.; Zachmann, H. G.; Polymer 1994, 35, 12.

[94] Graff, M. S.; Boyd, R. H.; Polymer 1994, 35, 1797.

[95] Nakayasu, H.; Markovitz, H.; Plazek. D. J.; Trans. Soc. Rheol. 1961, 5, 261.

[96] McCrum, N. G.; Morris, E. L.; Proc. R. Soc. (London) 1964, A281, 258.

[97] Hu, W.-G.; Boeffel, C.; Schmidt-Rohr, K.; Macromolecules 1999, 32, 1611.

[98] Boyd, R. H.; Polymer 1985, 26, 323.

[99] Boyd, R. H.; Polymer 1985, 26, 1123.

[100] Boyd, R. H.; Liu, F.; in "Dielectric Spectroscopy of Polymeric Materials", Runt, J. P.; Fitzgerald, J. J.; editors; American Chemical Society, Washington, DC 1997.

[101] Schmidt-Rohr, K.; Spiess, H. W.; Macromolecules 1991, 24, 5288.

[102] Zhou, H.; Wilkes, G. L.; Macromolecules 1997, 30, 2412.

[103] Mano, J. F.; Sousa, R. A.; Reis, R. L.; Cunha, A .M.; Bevis, M. J.; Polymer 2001, 42, 6187.

[104] Mano, J. F.; Macromolecules 2001, 34, 8825.

[105] Starkweather, H. W.; Macromolecules 1981, 14, 1277.

[106] Starkweather, H. W.; Macromolecules 1988, 21, 1798.

[107] Mano, J. F.; Lanceros-Méndez, S.; J. Appl. Phys. 2001, 89, 1844.

[108] "Polymer Liquid Crystals", Ciferri, A.; Krigbaum, W. R.; Meyer, R. B.; editors; Academic Press, Inc., New York 1982.

[109] Donald, A. M.; Windle, A. H.; "Liquid Crystalline Polymers", Cambridge University Press, Cambridge 1992. 
[110] Simon, G. P.; in "Dielectric Spectroscopy of Polymeric Materials", Runt, J. P.; Fitzgerald, J. J.; editor; American Chemical Society, Washington, DC 1997.

[111] Mano, J. F.; Gómez Ribelles, J. L.; Macromolecules 2003, 36, 2816.

[112] Mano. J. F.; J. Macromol. Sci. Phys. 2003, 42, 1125.

[113] Zentel, R.; Strobl, G. R.; Macromolecules 1985, 18, 960.

[114] Schönhals, A.; Wolff, D.; Springer, J.; Macromolecules 1998, 31, 9019.

[115] Mano, J. F.; Alves, N. M.; Meseguer Dueñas, J. M.; Gómez Ribelles J. L.; Polymer 1999, 40, 6545.

[116] Schick, C.; Sukhorukov, D.; Schönhals, A.; Macromol. Chem. Phys. 2001, 202, 1398.

[117] Mano, J. F.; Thermochimica Acta 1999, 332, 161.

[118] Kremer, F.; in "Dielectric Spectroscopy of Polymeric Materials", Runt, J. P.; Fitzgerald, J. J.; editors; American Chemical Society, Washington, DC 1997. 